ARTICLE

\title{
Genomic aberrations after short-term exposure to colibactin-producing E. coli transform primary colon epithelial cells
}

\author{
Amina Iftekhar (10 1, Hilmar Berger (10 1,2,3, Nassim Bouznad4 ${ }^{4}$, Julian Heuberger ${ }^{1,3}$, Francesco Boccellato (1) 1,5, \\ Ulrich Dobrindt 6 , Heiko Hermeking ${ }^{4,7,8}$, Michael Sigal (1) ${ }^{1,3,9 凶} \&$ Thomas F. Meyer (1) ${ }^{1,2 凶}$
}

Genotoxic colibactin-producing pks+ Escherichia coli induce DNA double-strand breaks, mutations, and promote tumor development in mouse models of colorectal cancer (CRC). Colibactin's distinct mutational signature is reflected in human CRC, suggesting a causal link. Here, we investigate its transformation potential using organoids from primary murine colon epithelial cells. Organoids recovered from short-term infection with pks+E. coli show characteristics of CRC cells, e.g., enhanced proliferation, Wnt-independence, and impaired differentiation. Sequence analysis of Wnt-independent organoids reveals an enhanced mutational burden, including chromosomal aberrations typical of genomic instability. Although we do not find classic Wnt-signaling mutations, we identify several mutations in genes related to p53-signaling, including miR-34a. Knockout of Trp53 or miR-34 in organoids results in Wnt-independence, corroborating a functional interplay between the p53 and Wnt pathways. We propose larger chromosomal alterations and aneuploidy as the basis of transformation in these organoids, consistent with the early appearance of chromosomal instability in CRC.

\footnotetext{
${ }^{1}$ Department of Molecular Biology, Max Planck Institute for Infection Biology, Berlin, Germany. ${ }^{2}$ Laboratory of Infection Oncology, Institute of Clinical Molecular Biology, Christian Albrechts University of Kiel and University Hospital Schleswig Holstein - Campus Kiel, Kiel, Germany. ${ }^{3}$ Department of Internal Medicine, Gastroenterology and Hepatology, Charité University Medicine, Berlin, Germany. ${ }^{4}$ Experimental and Molecular Pathology, Institute of Pathology, Ludwig Maximilians University, München, Germany. ${ }^{5}$ Ludwig Institute for Cancer Research, University of Oxford, Oxford, UK. ${ }^{6}$ Institute of Hygiene, University of Münster, Münster, Germany. ${ }^{7}$ German Cancer Consortium (DKTK), Partner Site München, München, Germany. ${ }^{8}$ German Cancer Research Center (DKFZ), Heidelberg, Germany. ${ }^{9}$ Berlin Institute for Medical Systems Biology, Max Delbrück Center for Molecular Medicine, Berlin, Germany.

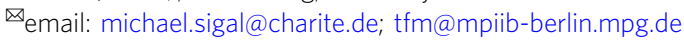


C olorectal cancer (CRC) is the second leading cause of cancer-related mortality worldwide. The genetic landscape of mutations that drive carcinogenesis is well explored and involves the Wnt, KRAS, TGF- $\beta$, and 553 pathways ${ }^{1}$. Combinations of mutations in these pathways are responsible for the transformation of epithelial cells ${ }^{2,3}$, and constitutively active Wnt signaling is considered to be a crucial feature of almost all CRCs ${ }^{4}$. Wnt signaling drives proliferation of colon cells and is physiologically active only in the stem cell compartment, while differentiated cells lack Wnt signaling 5 . Experimental and patient-derived data indicate that constitutively active Wnt signaling is an early event in the CRC cascade, often achieved by mutation of $A P C$ and subsequent loss of heterozygosity $(\mathrm{LOH})^{1,6}$. Although, this holds true for sporadic cases of CRC, cases driven by chronic inflammatory disorders, such as ulcerative colitis or Crohn's colitis, are associated with a distinct mutational profile, in which mutations in the p53 pathway are thought to occur as an early event ${ }^{7-10}$. While many of the mutations in driver genes are due to single nucleotide substitutions and small insertion and deletions, chromosomal aberrations are frequent in CRC and found in the majority of cases ${ }^{4}$. Chromosomal instability (CIN) is often attributed to cell-intrinsic causes like spindle assembly checkpoint defects $^{11}$, but the exact mechanisms leading to CIN are mostly unknown.

Recent data suggest that environmental factors are important drivers of CRC and that the colonic microbiota plays an important role in both the development and progression of $\mathrm{CRC}^{12-14}$. In addition to microbial factors that induce inflammation and therefore contribute to carcinogenesis by triggering oxidative stress or changes in the stem cell niche, certain bacteria that colonize the gut appear to directly affect the genomic integrity of epithelial cells through genotoxins. In this context, colibactin is a prominent genotoxin produced by several Enterobacteriaceae members, such as E. coli belonging to phylogenetic group B2. This toxin is a polyketide/nonribosomal peptide hybrid, encoded by the pks pathogenicity island. Infection of eukaryotic cell lines with $p k s+E$. coli was shown to cause DNA double-strand breaks (DSBs), leading to megalocytosis and cell cycle arrest ${ }^{15}$. Short exposure of an intestinal epithelial cell line to $p k s+E$. coli has been reported to cause genomic instability and anchorageindependent growth ${ }^{16}$. Significant enrichment of $p k s$ gene clusters has been observed in CRC metagenomes ${ }^{13}$. An increase in colonic mucosa-associated $E$. coli that possess the pks gene has been observed in inflammatory bowel disease (IBD), familial adenomatous polyposis (FAP), and CRC patients ${ }^{17-19}$. Colibactin alkylates DNA through covalent modifications and forms stable adenine-colibactin adducts in cultured mammalian cells and in vivo ${ }^{20-22}$. It has also been shown to leave a specific mutational signature in CRC patients ${ }^{23,24}$. Moreover, $p k s+E$. coli enhance tumorigenesis in different murine models of FAP and CRC, such as mice heterozygous for $A p c^{18}$, interleukin-10-deficient $\left(I l 10^{-/-}\right)$ germ-free mice ${ }^{17}$, and the azoxymethane/dextran sulfate sodium (AOM/DSS) model ${ }^{25}$. Notably, in these mouse models, tumors can also arise in the absence of colibactin, leaving it uncertain whether colibactin merely enhances tumorigenesis or whether it can also play a causative role. To study genomic instability caused by $p k s+E$. coli in vitro, infections have only been performed with transformed or immortalized cell lines, and it remains unclear whether healthy primary colon epithelial cells respond to infection in the same way.

To address this, we developed primary cell culture models derived from normal murine colon epithelial cells. We infected organoids as well as polarized monolayers derived from primary colon epithelial cells with $p k s+E$. coli or an isogenic mutant that is defective for colibactin synthesis. In these models, we were able to reproduce early responses to infection, including DSBs and development of megalocytosis-effects unique to strains with a functional $p k s$ island. Furthermore, we examined whether colibactin-induced DNA damage after short-term infection causes genomic instability, mutations, or changes in niche dependence of colon organoids. We specifically investigated Wntindependence due to its clinical relevance. Our findings reveal that the genotoxic effect of colibactin is sufficient to rapidly cause genomic aberrations in primary colon cells and to induce multiple features of transformation reminiscent of CRC. These transformed cells did not exhibit the typical colibactin mutational signature, suggesting that improper repair of colibactin-induced cross-links after short exposure to the bacteria can lead to copy number variations (CNV) and other mutations. Accordingly, chromosomal aberrations causing aneuploidy induced by colibactin are sufficient to cause primary cell transformation and precede detectable genomic imprinting of the colibactin mutational signature. The transforming capacity of colibactinproducing $E$. coli may thus be higher than what is estimated from the presence of the mutational signature alone.

\section{Results}

$p k s+E$. coli cause DNA damage, megalocytosis, and formation of multinucleated cells in primary colon epithelial cell cultures. To test whether the previously observed phenotypes of megalocytosis and DNA damage can be reproduced upon infection of human colon adenocarcinoma Caco-2 cells with the colibactinproducing ( $p k s+)$ wild-type (WT) M1/5 E. coli, we infected this cell line for $3 \mathrm{~h}$ at multiplicity of infection (MOI) 20. To ensure the effects were colibactin-specific, we used the E. coli $\Delta c l b R$ mutant strain ${ }^{26}$, which is defective for colibactin synthesis, as the negative control. Cells were immunostained for $\gamma \mathrm{H} 2 \mathrm{AX}$, an early response to DNA damage required for the recruitment of DNA damage repair proteins. We observed $\gamma \mathrm{H} 2 \mathrm{AX}$-positive cells, as well as megalocytosis and multinucleated cells in cultures infected with pks+ WT E. coli, while cultures infected with the mutant strain exhibited significantly less DNA damage (Fig. 1a). We next examined whether similar phenotypic changes are also observed after infection of primary cells. As it is difficult to image megalocytosis and multinucleated cells in organoids, we utilized mucosoids ${ }^{27}$, polarized epithelial monolayers grown in air-liquid interface $^{28}$. For this, mouse primary colon cells were first grown as conventional $3 \mathrm{D}$ organoids ${ }^{29}$, then sheared into single cells and seeded on collagen-coated polycarbonate filter inserts. Cultures in air-liquid interface form highly polarized monolayers, with secreted mucus accumulating on the apical side, similar to the epithelium in vivo (Fig. 1b). Immunolabeling of cultures shows that the cells express $\beta$-catenin and MUC2, the typical mucin of the colon (Fig. 1c, top). They became fully polarized, as indicated by basolateral E-cadherin staining and the presence of eccentric nuclei, and focally expressed Ki67, a marker of proliferating cells (Fig. 1c, bottom). After infection at MOI 5 for $3 \mathrm{~h}$, we detected $\gamma \mathrm{H} 2 \mathrm{AX}$-positive cells, megalocytosis, and multinucleated cells, similar to the phenotypes seen in Caco-2 cells (Fig. 1d). These phenotypes were not seen after infection with the $\Delta c l b R$ mutant. Thus, primary cells show a similar phenotypic response to colibactin as previously observed in cell lines.

We also infected murine colon organoids with $p k s+E$. coli at MOI 5 for $3 \mathrm{~h}$. Organoids were first broken up, so that both the basal and apical sides were exposed to the bacteria. Whole-mount immunofluorescence labeling was carried out 3 days later, after organoids had re-formed. Again, we observed $\gamma \mathrm{H} 2 \mathrm{AX}$-positive cells only after infection with the pks+ WT E. coli strain, indicating that this system is suitable for studying the effects of colibactin on primary cells (Fig. 1e). To confirm that the effects observed in the context of primary cell infection were not due to 


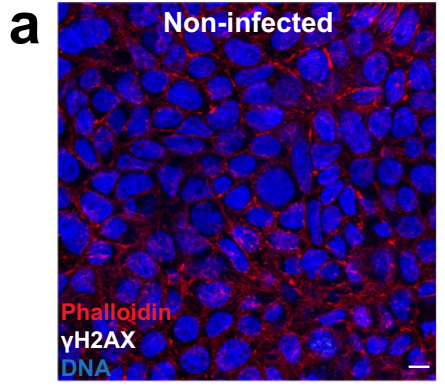

b

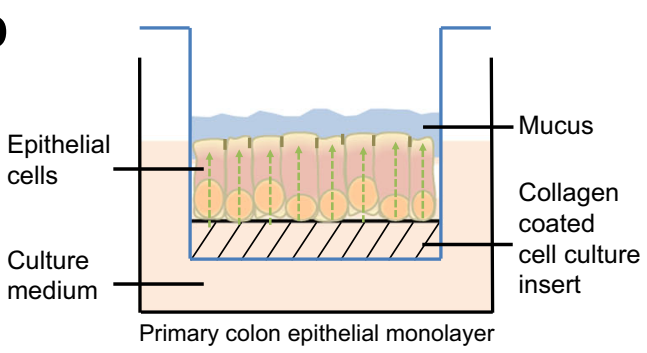

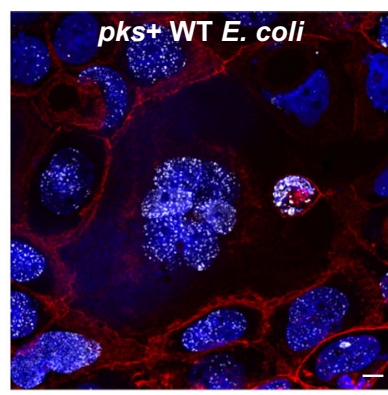

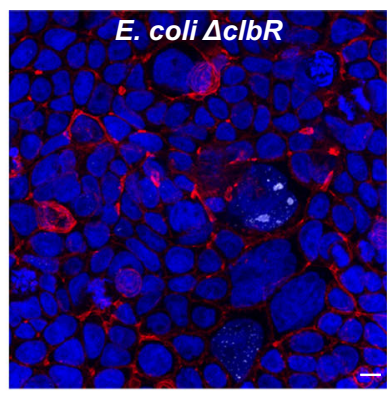

C
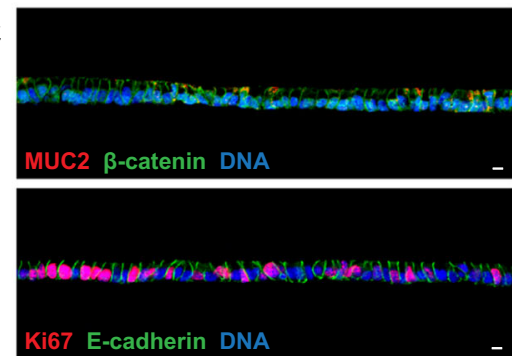

Ki67 E-cadherin DNA
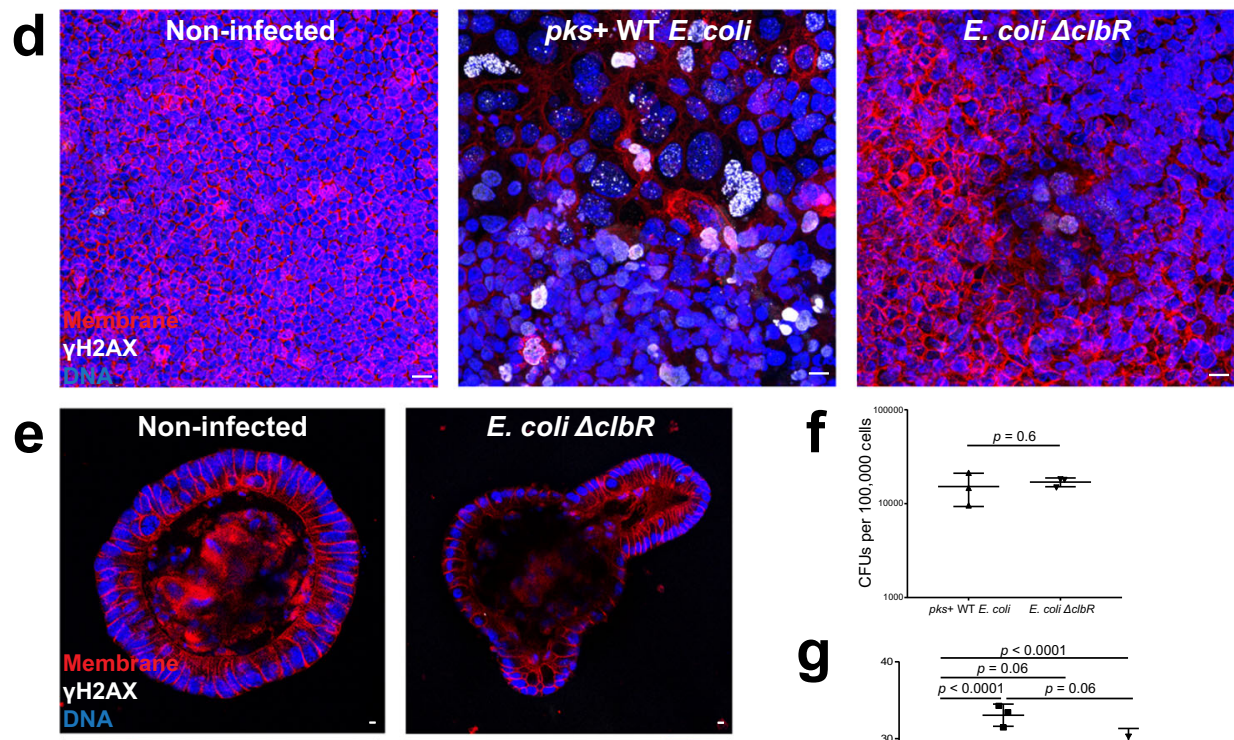

f

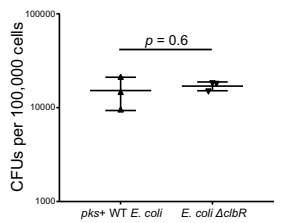

g

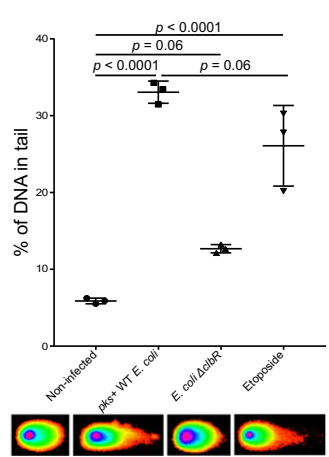

differences in infectivity between WT and $\Delta c l b R$ mutant E. coli, we performed colony-forming unit (CFU) analysis upon infection of organoids, confirming equal colonization rate (Fig. 1f).

To further confirm the presence of DSBs, we measured damaged DNA in murine colon organoid cells by neutral comet assay. The amount of DNA damage caused by infection with $p k s+$ WT E. coli was similar to that induced by treatment with etoposide, used as a positive control. The $\Delta c l b R$ mutant caused only minimal DNA damage compared to the non-infected condition (Fig. 1g). Infection of human colon organoids also resulted in $\gamma \mathrm{H} 2 \mathrm{AX}$-positive cells and caused significantly more DNA damage in neutral comet assay only in the pks+ WT E. coliinfected condition (Supplementary Fig. 1a, b). Furthermore, upon infection with pks+ WT E. coli, human colon organoids showed nuclear expression of the DSB repair proteins, phosphocheckpoint kinase 2 (Chk2) (Supplementary Fig. 2a), and p53binding protein 1 (53BP1) (Supplementary Fig. 2b), indicating that DSB repair is activated in response to colibactin. We therefore conclude that primary cells show DNA damage, megalocytosis, formation of multinucleated cells, and expression of DSB repair proteins upon exposure to $p k s+E$. coli, which is dependent on the synthesis of colibactin.

Wnt-independent growth of organoids after infection with $p k s+E$. coli. Although DSBs are known to cause apoptosis or cellular senescence, immortalized Chinese hamster ovary cells 


\section{Fig. 1 pks + E. coli cause DNA damage, megalocytosis, and formation of multinucleated cells in primary colon epithelial cell cultures.}

a Immunofluorescence staining of Caco-2 cells showing $\gamma \mathrm{H} 2 \mathrm{AX}$ (white). Phalloidin (red) stains for actin filaments and Hoechst (blue) stains for DNA. Caco-2 cells infected with pks+ WT E. coli are $\gamma \mathrm{H} 2 \mathrm{AX}$-positive, display megalocytosis, and are multinucleated. Less $\gamma \mathrm{H} 2 \mathrm{AX}$ is detected in cells infected with the mutant strain ( $E$. coli $\Delta c l b R$ ) defective for colibactin synthesis. Image representative of three independent replicates. Scale bars: $10 \mu \mathrm{m}$. $\mathbf{b}$ Epithelial cells from colon organoids are sheared and seeded on collagen-coated polycarbonate filter inserts. The cells attach and form a columnar polarized monolayer. Secreted mucus accumulates on the apical side. c Top: Cross-section of the polarized monolayer showing expression of colonic mucus MUC2 (red) and $\beta$ catenin (green). Hoechst (blue) stains for DNA. Bottom: Cross-section of the polarized monolayer showing expression of basolateral E-cadherin (green) and the proliferation marker Ki67 (red). Hoechst (blue) stains for DNA. Image representative of three independent replicates. Scale bars: $10 \mu \mathrm{m}$.

d tdTomato (red) expressing colonic monolayer immunostained for $\gamma \mathrm{H} 2 \mathrm{AX}$ (white); Hoechst (blue) stains for DNA. Megalocytosis, multinucleated cells, and $\gamma \mathrm{H} 2 \mathrm{AX}$-positive cells are observed in monolayer infected with pks+ WT E. coli. Image representative of three independent replicates. Scale bars: $10 \mu \mathrm{m}$. e Murine colon organoids expressing membrane tdTomato (red) immunostained for $\gamma \mathrm{H} 2 \mathrm{AX}$ (white); Hoechst (blue) was used to stain for DNA. Cells in colon organoids are $\gamma \mathrm{H} 2 \mathrm{AX}$-positive only when infected with the pks+ WT E. coli. Image representative of three independent replicates. Scale bars: $10 \mu \mathrm{m}$. f CFUs per 100,000 cells of murine colon organoids from three independent replicates. Data represent mean $\pm \mathrm{SD}, p<0.05$, as calculated by two-sided unpaired Student's $t$-test. $\mathbf{g}$ Comet assay of murine colon organoid cells (each data point represents the mean of $>80$ cells) from three independent replicates shows more DNA damage in pks+ WT E. coli-infected cells, which is comparable to etoposide, the positive control. Data represent mean \pm SD, $p<0.05$, as calculated by one-way ANOVA and Tukey's test. Source data are provided as a Source Data file.

have been shown to continue to proliferate in the presence of DNA damage after infection with $p k s+E$. coli ${ }^{16}$. We wanted to explore the response of non-immortalized primary cells equipped with normal DNA repair mechanisms. Primary polarized epithelial monolayers were infected with $p k s+E$. coli and immunolabeled for $\gamma \mathrm{H} 2 \mathrm{AX}$ and Ki67. After $3 \mathrm{~h}$ of infection, several cells were double-positive for $\gamma \mathrm{H} 2 \mathrm{AX}$ and Ki67, indicating that they proliferated despite DNA damage. In contrast, monolayers that were treated with cisplatin, a chemotherapeutic agent known to cross-link DNA and form cytotoxic adducts similar to colibactin $^{30}$, contained no double-positive cells (Supplementary Fig. 3a). Immunofluorescence also showed a lower expression of $\gamma \mathrm{H} 2 \mathrm{AX}$ for cisplatin-treated cells, which was compatible with the neutral comet assay results (Supplementary Fig. 3b).

Cell proliferation in the presence of incompletely repaired DNA damage can cause somatic mutations, which could potentially lead to independence from niche factors. Such niche escape is a hallmark of cancer organoids ${ }^{2,3}$. To assess their organoid-forming capacity, we infected organoids at MOI 5 for 3 $\mathrm{h}$, re-seeded them in Matrigel and treated them with gentamicin to kill the bacteria. To determine the extent of cell death, we performed an organoid-forming assay where 50,000 cells were seeded in each condition. We observed that the organoid-forming efficiency for $p k s+$ WT E. coli-infected organoids was reduced by about $40 \%$, but there was no difference between WT and $\Delta c l b R$ mutant E. coli-infected conditions (Fig. 2a). As the earliest and most frequently observed mutations in CRC are ones that lead to constitutive Wnt pathway activation, at the next passage we reseeded the organoids into complete 3D medium, which contains both Wnt and the Wnt agonist CHIR99021, as well as into medium lacking these two factors (Fig. 2b). We noticed that noninfected and $\triangle c l b R$ mutant-infected organoids failed to re-grow in Wnt-free medium, while in the pks+ WT E. coli-infected condition a few organoids did re-form (Fig. 2c). These Wntindependent organoids could be further passaged and expanded. Thus, a short exposure of primary cells to colibactin-producing $E$. coli was sufficient to cause somatic adaptation that led to the generation of Wnt-independent clones.

We also performed experiments with E. coli Nissle 1917, which is widely used as a probiotic and known to harbor the $p k s$ island. Neutral comet assay demonstrated that Nissle 1917 caused fewer DSBs compared to the M1/5 strain, but more than was seen in the non-infected and the E. coli Nissle $1917 \Delta c l b B$ mutant-infected conditions (Supplementary Fig. 4a). Furthermore, we analyzed the cross-linking capacity of these two strains. WT bacteria were added to $1.5 \mu \mathrm{g}$ of pfdA8 plasmid DNA at CFUs ranging from $1^{*} 10^{6}$ to $10^{*} 10^{6}$. A single CFU of $120^{*} 10^{6}$ was added for the mutant strains. A band at $3.4 \mathrm{~kb}$ indicates the presence of crosslinked DNA. Although both strains are commensal E. coli, the M1/5 strain showed a higher amount of cross-linked DNA compared to the Nissle 1917 strain. The absence of a band at 3.4 $\mathrm{kb}$ for the mutant strains indicated that they did not cross-link DNA (Supplementary Fig. 4b). Upon infection of murine colon organoids, no Wnt-independent organoids were observed in the E. coli Nissle 1917-infected condition, whereas the E. coli M1/ 5 strain once again generated Wnt-independent organoids. Additionally, treatment with etoposide or cisplatin also did not generate Wnt-independent organoids (Supplementary Fig. 4c). Therefore, the genotoxicity varies between strains of $p k s+E$. coli and also as compared to other DNA damaging agents.

Wnt-independent organoids exhibit increased organoid formation capacity, higher proliferation, and longevity. To characterize the consequences of infection on organoid growth, we measured the organoid-forming capacity of non-infected, $p k s+$ WT E. coli-infected and $\triangle c l b R$ mutant-infected organoids as well as the Wnt-independent organoids generated in the previous experiment. After dissociation, 10,000 single cells were re-seeded from each condition, and the number of organoids formed was quantified. Cells that were obtained from the WT pks+E. coliinfected condition had a higher organoid-forming capacity compared to those that were non-infected or infected with the $\triangle c l b R$ mutant. However, the highest capacity was observed for cells derived from the Wnt-independent organoids (Fig. 2d, e). The Wnt-independent organoids were also found to grow for a longer period than non-infected organoids, which could only be maintained for about 20 passages. All biological replicates of Wnt-independent organoids were maintained in culture for more than 1 year, with one replicate maintained for over 2.5 years, and none showed any sign of slowed growth when the culture was discontinued. The passaging ratio was also higher for the Wntindependent organoids, reflecting their increased growth capacity (Fig. 2f). Accordingly, immunolabeling of organoids 7 days after passaging showed that Wnt-independent organoids had a higher percentage of Ki67-positive cells when compared to non-infected organoids (Fig. $2 \mathrm{~g}$ and $\mathrm{h}$ ). In summary, Wnt-independent colon organoids generated by the action of colibactin had increased organoid-forming capacity, an increased number of proliferating cells, and potentially unlimited expansion capacity in culture.

Wnt-independent organoids show upregulated Wnt/ $\beta$-catenin signaling and downregulation of differentiation genes. Next, we determined if stem cell-associated pathways were upregulated 

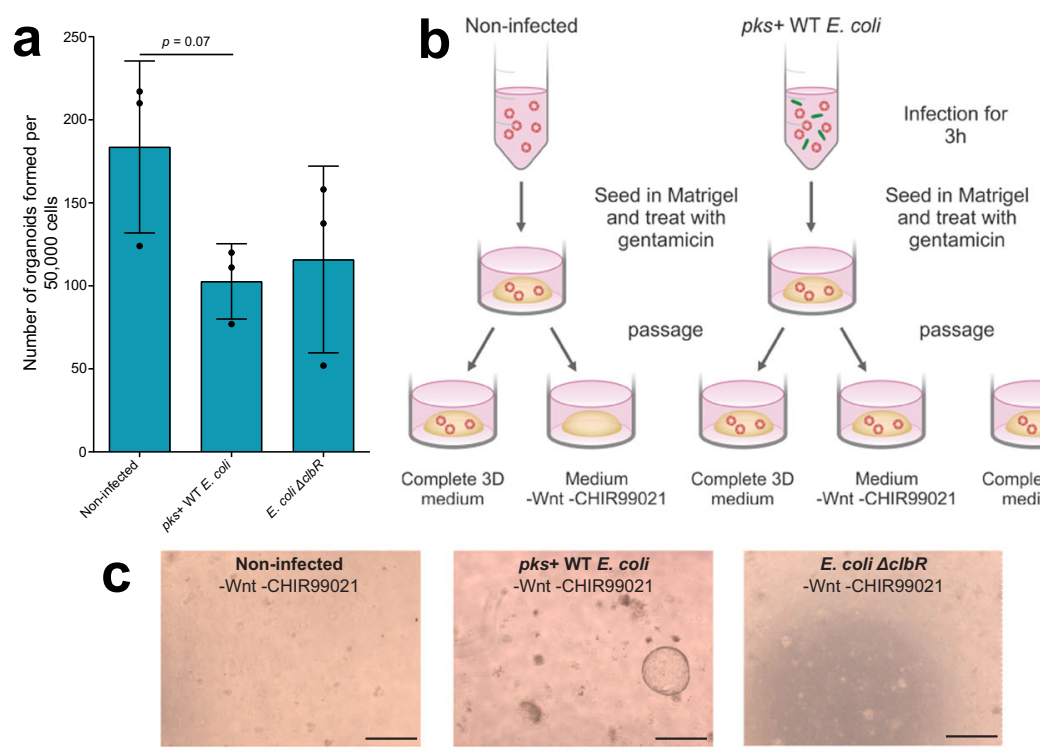

E. coli $\triangle c l b R$
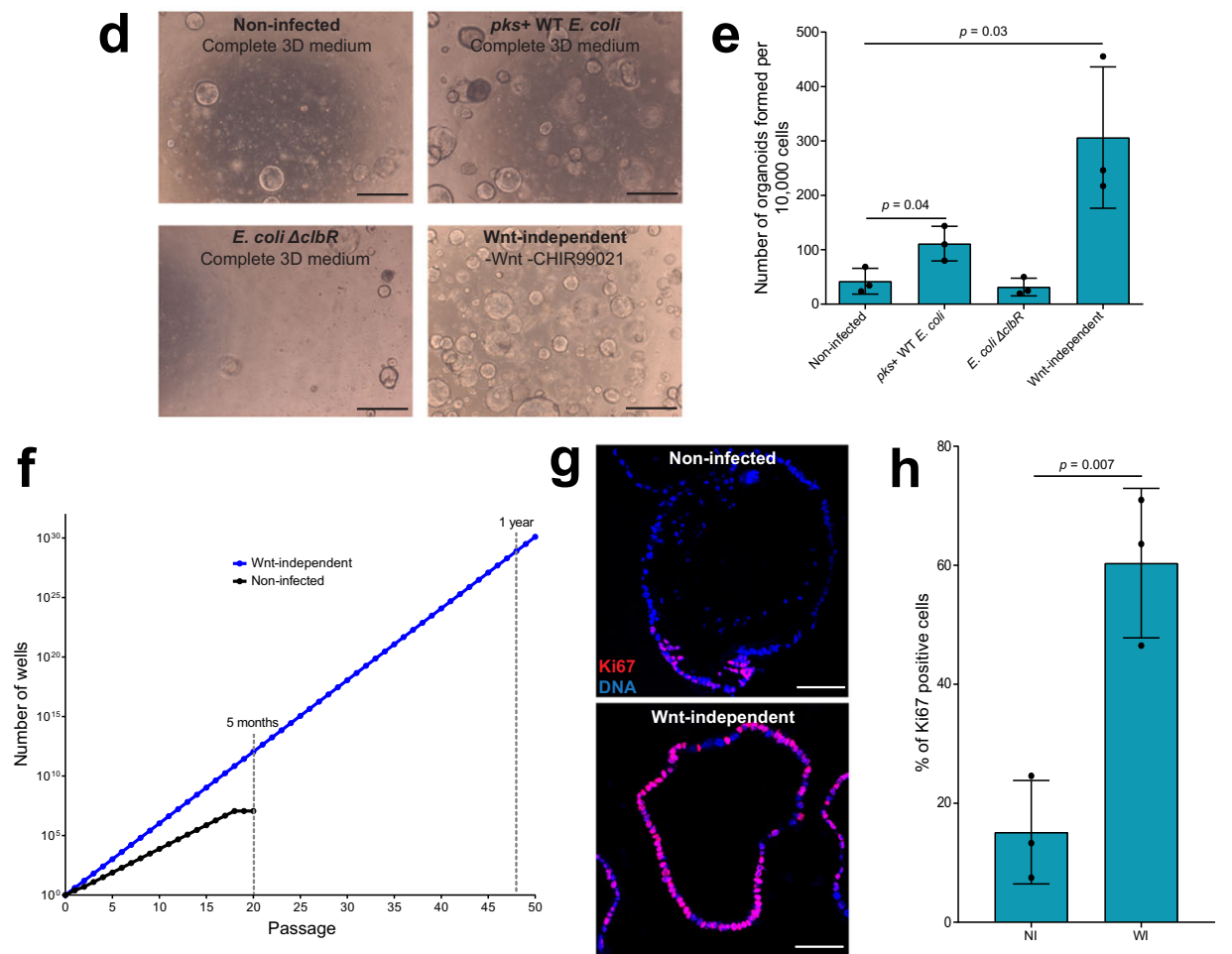

Fig. 2 Wnt-independent growth of organoids after infection with pks + E. coli. a Organoids formed after seeding 50,000 non-infected, pks + WT E. coliinfected, and $E$. coli $\Delta c l b R$-infected cells from three independent replicates. Data represent mean $\pm S D, p<0.05$, as calculated by two-sided unpaired Student's t-test. b Scheme describing the infection of organoids. c Organoids grow without the addition of Wnt and CHIR99021 to the medium for the pks+ WT E. coli-infected condition, but not for the non-infected or E. coli $\Delta c l b R$-infected conditions. Image representative of four independent replicates. Scale bars: $1 \mathrm{~mm}$. d Organoids formed after seeding 10,000 cells in each condition. Image representative of three independent replicates. Scale bars: $1 \mathrm{~mm}$. e Quantification of the number of organoids formed after seeding 10,000 cells in each condition from three replicates. Data represent mean \pm SD, $p<0.05$, as calculated by two-sided unpaired Student's $t$-test. $\mathbf{f}$ Wnt-independent organoids have a higher splitting ratio and grow indefinitely when compared to non-infected organoids. Graph representative of four independent replicates. $\mathbf{g}$ Immunofluorescence staining for Ki67 (red) in non-infected organoids and Wnt-independent organoids. Hoechst (blue) stains for DNA. Image representative of three independent replicates. Scale bars: $100 \mu \mathrm{m}$. $\mathbf{h}$ Quantification of the percentage of Ki67-positive cells from three replicates. Data represent mean $\pm \mathrm{SD}, p<0.05$, as calculated by two-sided unpaired Student's $t$-test. $\mathrm{NI}=$ non-infected and $\mathrm{WI}=$ Wnt-independent. Source data are provided as a Source Data file.

in Wnt-independent organoids. We performed RNA sequencing of non-infected and Wnt-independent organoids. All cultures were grown in a complete 3D medium for 5 days, followed by growth in medium without Wnt and CHIR99021 for 3 days. GSEA revealed a significant enrichment of Lgr5 signature genes ${ }^{31}$ in Wnt-independent organoids when compared to non-infected organoids (Fig. 3a). All three replicates of Wnt-independent organoids expressed higher levels of Wnt target genes when compared to the respective non-infected cultures (Fig. 3b). We performed RT-qPCR for Lgr5 (the classic intestinal stem cell marker), Lef-1, Fzd7, and $\beta$-catenin (Ctnnb1) and found higher expression of these genes in Wnt-independent organoids 


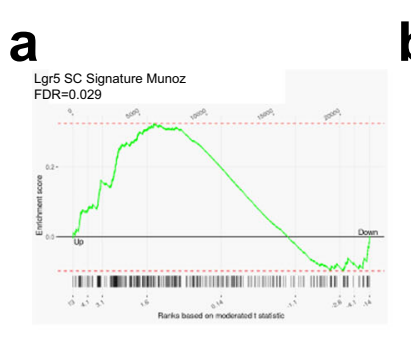

Non-infected organoids + Wnt medium

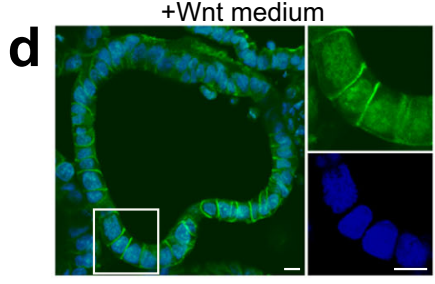

-Wnt medium for $24 \mathrm{~h}$

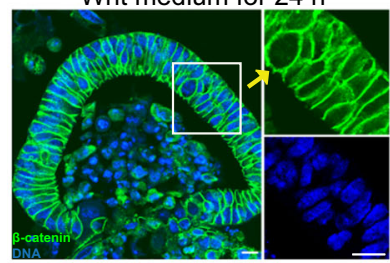

f

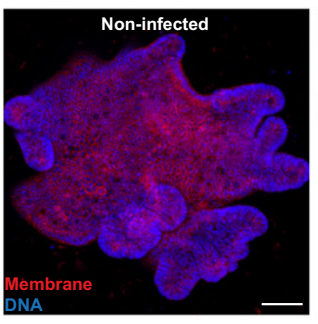

h

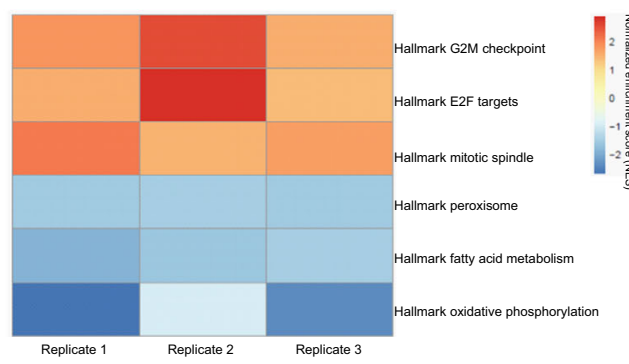

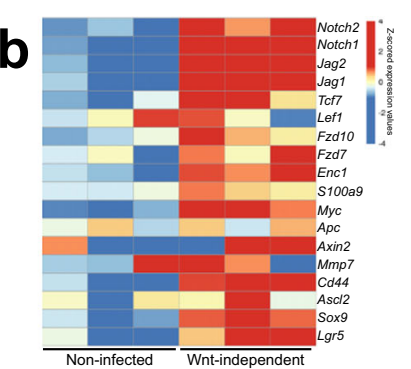

Wnt-independent organoids + Wnt medium

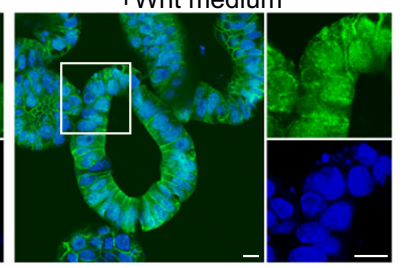

Continuously -Wnt medium

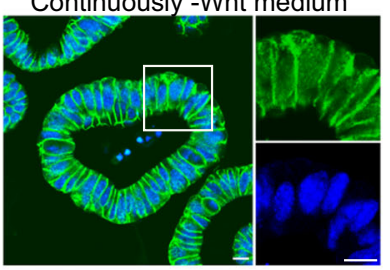

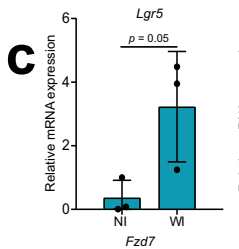
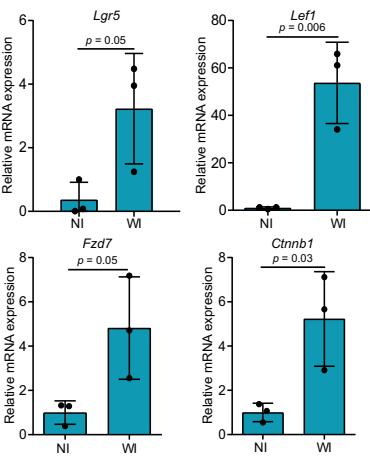

e
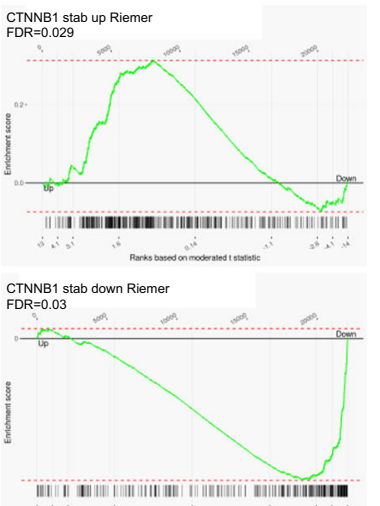

g
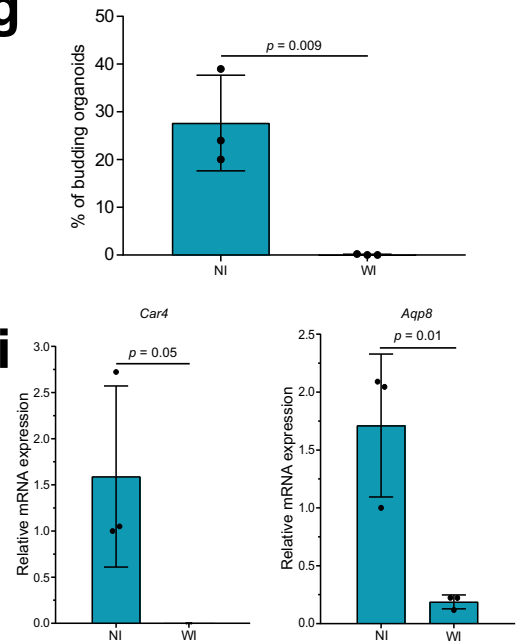

(cultured in - Wnt medium) when compared to non-infected organoids (cultured in + Wnt medium) (Fig. 3c). Immunostaining for $\beta$-catenin showed the presence of nuclear $\beta$-catenin in Wnt-independent organoids in the presence or absence of Wnt, indicating active $\mathrm{Wnt} / \beta$-catenin signaling. Non-infected organoids kept in the absence of Wnt for $24 \mathrm{~h}$ showed absence of nuclear $\beta$-catenin in several nuclei (Fig. 3d). Furthermore, gene sets implicated in Wnt signaling that are upregulated or downregulated due to stabilization of $\beta$-catenin in intestinal organoids ${ }^{32}$ showed a significant positive and negative enrichment, respectively, in Wnt-independent organoids when compared to non-infected organoids (Fig. 3e). The Wnt-independent organoids rely on endogenous Wnt production because treatment with a porcupine inhibitor (IWP-2) led to inhibition of their growth, which could be rescued by exogenous Wnt (Supplementary Fig. 5a). This indicated that these organoids showed a higher sensitivity to endogenous Wnt. We explored the expression pattern of selected genes encoding for Wnt receptors and found a higher expression of $\operatorname{Lrp} 6, \mathrm{Fzd7}$, and Fzd10, which could be responsible for the increased sensitivity to endogenous Wnt signals (Fig. 3b, c and Supplementary Fig. 5b).

Upregulated Wnt signaling in intestinal organoids has previously been shown to cause cells to adopt a proliferative progenitor phenotype, which results in a shift from the asymmetric crypt-villus architecture to a spheroid, cyst-like morphology devoid of differentiated cell types ${ }^{33}$. Accordingly, we noticed that Wnt-independent organoids formed spheroid, cyst-like organoids, unlike the non-infected condition (Fig. 3f). To quantify this, all conditions were kept in complete 3D medium for 7 days, and any organoid showing a budding structure was scored as a differentiated organoid (Fig. 3g). Further, GSEA revealed an upregulation of gene sets implicated in proliferation and downregulation of gene sets involved in fatty acid metabolism, which are active in differentiated colonocytes 
Fig. 3 Wnt-independent organoids show upregulated Wnt/ק-catenin signaling and downregulation of differentiation genes. a Global comparison using gene set enrichment analysis between intestinal stem cell genes ${ }^{31}$, and RNA sequencing results from three replicates. Genes are ordered according to their differential expression in Wnt-independent vs. non-infected samples from left to right. Intestinal stem cell genes are marked by black bars below the plot. Plot line shows the running enrichment score. $\mathbf{b}$ Wnt-independent organoids have a higher expression of Wnt target genes when compared to respective non-infected conditions. Data are mean-centered and averaged expression values for each replicate $(n=3)$ and condition. c RT-qPCR data showing expression of $L g r 5$, Lef1, Fzd7, and $\beta$-catenin (Ctnnb1) relative to Gapdh from three independent replicates. Data represent mean \pm SD, $p<0.05$, as calculated by two-sided unpaired Student's $t$-test. $\mathrm{NI}=$ non-infected and $\mathrm{WI}=$ Wnt-independent. $\mathbf{d}$ Immunostaining for $\beta$-catenin (green) in non-infected and Wntindependent organoids. Upon removal of Wnt from the medium for $24 \mathrm{~h}$, non-infected organoids show the absence of nuclear $\beta$-catenin (marked by the yellow arrow). DAPI (blue) stains for DNA. Image representative of three independent replicates. Scale bars: $10 \mu \mathrm{m}$. e Global comparison using gene set enrichment analysis between genes upregulated and downregulated during activated $\beta$-catenin signaling 32 , and RNA sequencing results from three replicates. Genes are ordered according to their differential expression in Wnt-independent vs. non-infected samples from left to right. Genes are marked by black bars below the plot. Plot line shows the running enrichment score. $\mathbf{f}$ Immunofluorescence image of a single organoid on day 7 after seeding. Image representative of three independent replicates. Scale bars: $100 \mu \mathrm{m}$. $\mathbf{g}$ Bar graph of the percentage of differentiated organoids from three replicates on day 7 after seeding. $>90$ organoids counted per condition for each replicate. Data represent mean \pm SD, $p<0.05$, as calculated by two-sided unpaired Student's $t$ test. $\mathrm{NI}=$ non-infected and $\mathrm{WI}=$ Wnt-independent. $\mathbf{h}$ Hallmark genes associated with mitosis (Hallmark G2M checkpoint, Hallmark E2F targets, and Hallmark mitotic spindle gene sets) are enriched among upregulated genes, and hallmark genes associated with differentiation (Hallmark peroxisome, Hallmark fatty acid metabolism, and Hallmark oxidative phosphorylation gene sets) are enriched among downregulated genes in all three replicates of Wnt-independent organoids when compared to non-infected organoids. Normalized enrichment score (NES) for a given gene set is the enrichment score normalized to mean of the enrichment scores for all data set permutations. Results are significant (FDR $<20 \%$ ), except for Hallmark oxidative phosphorylation of replicate 2. i RT-qPCR data showing expression of Car4 and Aqp8 relative to Gapdh from three independent replicates. Data represent mean $\pm S D, p<0.05$, as calculated by two-sided unpaired Student's $t$-test. $\mathrm{NI}=$ non-infected and $\mathrm{WI}=$ Wnt-independent. Source data are provided as a Source Data file.

(Fig. 3h). In addition, the enterocyte-specific differentiation genes carbonic anhydrase 4 (Car4) and aquaporin 8 (Aqp8) were downregulated in Wnt-independent organoids (Fig. 3i). Thus, infection-induced Wnt-independence of organoids was accompanied by a proliferative cell phenotype and impaired differentiation.

Wnt-independent organoids have major chromosomal aberrations and high mutational load. To determine whether the alterations induced by colibactin are linked to mutations, we generated clonal and pooled cultures from non-infected and Wnt-independent organoids and performed whole-genome (WGS) and whole-exome sequencing (WXS) on cells obtained from two replicates. We identified a higher number of clonal (allelic proportion >25\%) single nucleotide variants (SNV), insertion and deletion (indel) mutations, and detected rearrangement breakpoints (rearrangements) in Wnt-independent organoids compared to non-infected controls. Approximately 875 million and 650 million base pairs were affected by copy number variations $(\mathrm{CNV})$ in clones generated from Wntindependent organoids from replicate 1 and replicate 2, respectively (Fig. 4a). However, many of the identified mutations were shared between clones from the same mouse (genomic losses on chromosomes 4, 5, and 13 in replicate 1 and chromosomes 2, 4, 5, $10,13,17$, and 19 in replicate 2), indicating that they may have originated from the same mutated cell (Supplementary Fig. 6a). Thus, the Wnt-independent cells show features of CIN with $\mathrm{CNV}$, including predominant losses of whole chromosomes or chromosome arms (Fig. 4b), in agreement with chromosomal aberrations induced during mitosis ${ }^{16}$. Subclonal changes such as additional breaks, genomic gains, and genomic losses indicate ongoing instability, including chromosome 14 of replicate 2, which exhibited multiple breakpoints and $\mathrm{CNV}$ in close proximity with pure intrachromosomal relocations, which may be a sign of chromothripsis ${ }^{34}$. Over 3600 genes were found to be affected, mostly by CNV, but to a lesser extent by SNV or by both SNV and CNV. Copy-number imbalances affected about 3500 expressed protein-coding genes in each sample, while only about 10-20 genes showed protein changes due to single nucleotide substitutions or small indels (Fig. 4c). Among them were genes known to be involved in Wnt signaling, in the p53 pathway, and known cancer drivers (Fig. 4d). We did not identify CNV or known driver mutations in the mouse homologs of the key Wntsignaling genes $A p c$ or Ctnnb1. Instead, Wnt-independent clones showed copy number changes in a number of additional genes frequently mutated across cancers ${ }^{35}$ or specifically in colorectal carcinoma $^{4}$ (Supplementary Fig. 6b). Both replicates had a heterozygous loss of AT-rich interactive domain-containing protein $1 \mathrm{~A}$ (Arid1a), which is mutated in several types of cancer ${ }^{36}$. Aridla has been shown to act as a tumor suppressor in the mouse colon by enhancer-mediated gene regulation ${ }^{37}$, and in ovarian cancer ARID1A mutation has been shown to functionally inactivate $T P 53^{38}$. In both replicates of exome sequencing, there was a heterozygous loss of the micro-RNA-34a ( $m i R-34 a)$ gene. $m i R$ $34 a$ expression is induced by $\mathrm{p} 53$ and is known to play important roles in the downregulation of the Wnt pathway and in tumor suppression ${ }^{39,40}$. In one of our replicates, there was a heterozygous loss of cyclin-dependent kinase inhibitor 1a (Cdkn1a/p21) gene, while the other one showed a loss of cyclin-dependent kinase inhibitor $1 \mathrm{~b}(C d k n 1 b)$, both of which are involved in cell cycle arrest. Cdkn1a is a target of p53 and acts downstream to inhibit cellular proliferation. In both replicates, there was also a heterozygous loss of cyclin-dependent kinase inhibitor 2a $(C d k n 2 a)$, known to act upstream of p53. One replicate had gain-of-function of cyclindependent kinase $2(C d k 2)$ and cyclin-dependent kinase $4(C d k 4)$, genes involved in cell cycle progression that are negatively regulated by $\mathrm{p} 53$. The Wnt and $\mathrm{p} 53$ pathways are complex regulatory networks, therefore it is not unlikely that a mutation or a combination of mutations induced by colibactin disrupted these critical pathways, which are important for cellular proliferation and tumorigenesis.

A mutational signature of colibactin action on DNA has recently been identified ${ }^{23,24}$. We tested if we could identify hallmarks of this signature in our Wnt-independent clones. We neither identified the previously described increase in $\mathrm{T}>\mathrm{N}$ substitutions at ATA, ATT, and TTT $^{24}$ (Supplementary Fig. 6c) nor a relevant increase in small indels at A:T homopolymers (Supplementary Fig. 6d). We also did not observe an enrichment of structural variant breakpoints at the reported AAWWTT motif $^{23}$ (Supplementary Fig. 6e). Based on previous data ${ }^{24}$, only a small number of colibactin-associated SNV and small indels are expected in our experimental setting (i.e., about $4 \mathrm{SNV}$ and 1 indel after $3 \mathrm{~h}$ infection compared to about 500 additional single 


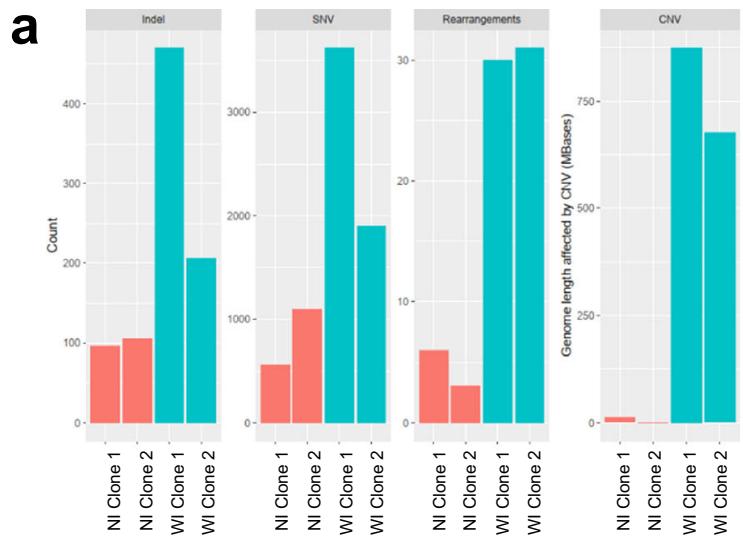

Replicate 1 -

NI (Non-infected) Clone 1

WI (Wnt-independent) Clone 1

Replicate 2 -

$\mathrm{NI}$ (Non-infected) Clone 2

WI (Wnt-independent) Clone 2

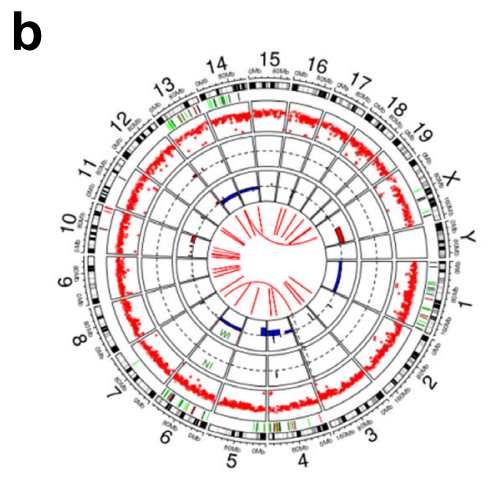

Replicate 1

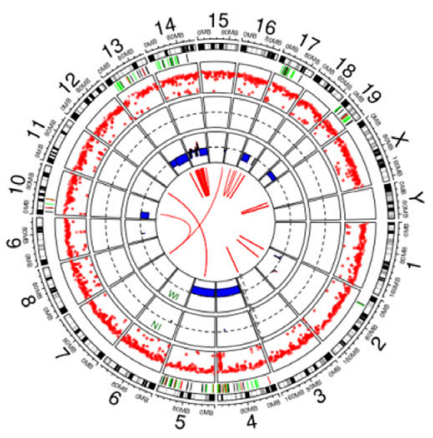

Replicate 2

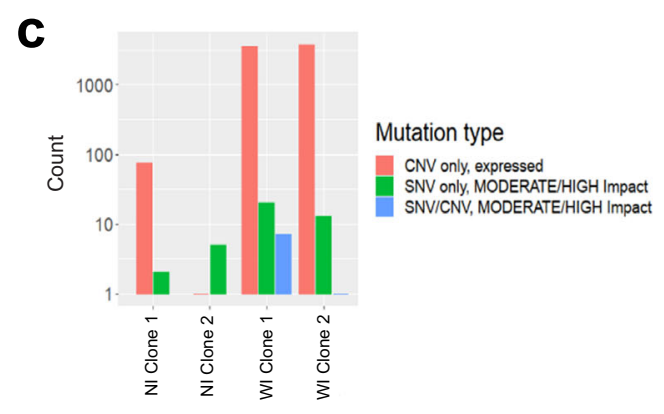

Fig. 4 Wnt-independent organoids have major chromosomal aberrations and high mutational load. a The number of detected insertions and deletions (indels), somatic single nucleotide variants (SNV), and detected rearrangement breakpoints (Rearrangements) in Wnt-independent (WI) and non-infected (NI) organoids in two independent replicates from whole-genome sequencing (WGS). Genome length affected by copy number variations (CNV) for Wntindependent $(\mathrm{WI})$ and non-infected (NI) organoids in two independent replicates from WGS. Each condition was compared to the pool of non-infected organoids of that respective experiment. b Circos plots showing CNV, SNV, indels, structural variants, and mutated genes in the Wnt-independent organoids for replicates 1 and 2 from WGS. Each section represents chromosomes 1-19, chromosome X, and chromosome Y. The central part shows aberrant genomic fusions in the Wnt-independent condition. The two innermost circles ( WI and NI) represent CNV in non-infected (NI) organoid clones and Wnt-independent (WI) organoid clones, respectively. The blue (genomic loss) and red (genomic gain) regions represent CNV. All losses of chromosomal regions are heterozygous. Each condition was compared to all the non-infected organoids of that respective replicate. A rainfall plot (red dots) shows the SNV and indel variations and the distances between them on the $y$-axis only for the Wnt-independent condition. The green, orange, and black lines depict positions of mutated Wnt pathway genes, known cancer driver genes, and p53 pathway genes, respectively, in the Wnt-independent condition. c The number of genes affected in each sample by CNV and/or SNV analyzed by WGS. For CNV, only genes expressed in non-infected controls were considered. For SNV, only mutations that change the amino acid sequence are shown. $\mathbf{d}$ Mutational status of genes of the Wnt pathway that are frequently affected in CRC, genes of the micro-RNA-34 family, and Trp53. Blue, genomic loss; red, genomic gain. Source data are provided as a Source Data file. 
base substitutions and 150 indels after 5 repetitions of 3 days of infection $^{24}$, assuming a uniform rate of mutations over time). On the other hand, chromosomal aberrations have been observed 24 $\mathrm{h}$ after a $4 \mathrm{~h}$ exposure to colibactin-producing $E$. coli ${ }^{16}$. Indeed, our data indicate that colibactin-induced cross-links might lead to genomic instability even after short contact, without evidence of a mutational signature, which most likely requires long-term or repetitive exposure to colibactin.

These sequencing results, therefore, show the genomic lesions in primary colon cells resulting from a single $3 \mathrm{~h}$ infection with $p k s+E$. coli. We conclude that Wnt-independent organoids show hallmarks of CIN and an increased mutational load affecting large numbers of genes, some of which are tightly linked to the p53 signaling pathway, which individually or in combination could have led to the development of niche independence.

The Trp53/miR-34 axis is disrupted in the Wnt-independent organoids. As we noticed that several genes involved in p53 signaling were affected in the Wnt-independent organoids upon exposure to $p k s+$ WT E. coli, we immunolabeled the organoids for p53 and observed the presence of nuclear p53 in several cells (Fig. 5a). We next checked the sensitivity of Wntindependent organoids to Nutlin-3a, which activates the p53 pathway and acts as an apoptosis inducer. While non-infected organoids did not grow when Nutlin-3a was added to the medium, the Wnt-independent organoids continued to grow (Fig. 5b), indicating a defect downstream of p53.

To examine if there is a connection between Wntindependence and disruption of the p53 pathway, we first tested whether loss of the Trp53 gene results in organoids that grow in the absence of Wnt. For this, we generated organoids from the colon of $\operatorname{Trp} 53^{\text {flox/flox }}$ mice. The Trp53 gene was floxed out by the transduction of organoids with an adeno-associated virus encoding the Cre recombinase (AAV-Cre). To select cells with a successful knockout, the organoids were grown in the presence of Nutlin-3a. The depletion of p53 was further verified by western blot analysis. Non-transduced organoids were grown for 6 days and then kept in the presence of Nutlin-3a for 2 days to stabilize the p53 protein, while transduced organoids were cultured with Nutlin-3a for 8 days, confirming the loss of $\operatorname{Trp} 53$ (Fig. 5c). The Trp53 KO organoids continued to grow when Wnt and CHIR99021 were withdrawn from the medium, while nontransduced organoids grew neither in the presence of Nutlin-3a nor in the absence of Wnt (Fig. 5d). Therefore, loss of Trp53 is sufficient to confer independence from extrinsic Wnt signals.

As we did not detect a mutation in the $\operatorname{Trp53}$ gene in the sequencing results, and as p53 was found in the nuclei, we hypothesized that Wnt-independence could be driven by an altered effector downstream of $\mathrm{p} 53$, and investigated if the loss of the $m i R-34 a$ gene could have led to extrinsic Wnt-independence in the organoids. $m i R-34 a$ is expressed at higher levels than $m i R$ $34 \mathrm{~b} / \mathrm{c}$, with the exception of the lung, in which $m i R-34 \mathrm{~b} / \mathrm{c}$ is highly expressed $^{41}$. RT-qPCR for pri-miR-34 showed downregulation of pri-miR-34a and pri-miR-34b/c in Wnt-independent organoids when compared to non-infected organoids (Fig. 5e). Lower expression of mature miR-34a and miR-34b, but not miR-34c, was observed in Wnt-independent organoids (Fig. 5f). It has previously been shown that some epithelial-mesenchymal transition (EMT)-inducing transcription factors can bind to E-boxes of $m i R-34 a$ and $m i R-34 b / c$ promoters, thereby repressing $m i R-34 a$ and $m i R-34 b / c$ expression ${ }^{42}$. GSEA of the Wnt-independent organoids also showed an enrichment of EMT-related genes (Supplementary Fig. 7a).

To explore the function of miR-34, we generated colon organoids from $m i R-34 a, m i R-34 b / c$, and $m i r-34 a / b / c$ KO mice, and found an increased expression of Lgr5 and $\beta$-catenin (Ctnnb1) in miR-34a KO organoids, which was even more pronounced in $m i R-34 a / b / c$ KO mouse-derived organoids. $m i R$ $34 a \mathrm{KO}$ and $m i R-34 a / b / c \mathrm{KO}$ organoids also showed increased expression of Lrp6 and Fzd7, similar to the Wnt-independent organoids generated upon infection (Supplementary Fig. 7b). Furthermore, we observed that colon organoids generated from $m i R-34 a / b / c$ KO mice were able to grow in the absence of Wnt and CHIR99021, whereas depletion of $m i R-34 a$ alone was insufficient to maintain Wnt-independent long-term cultures (Fig. 5g). Thus, we conclude that the heterozygous loss of the $m i R-34 a$ gene and the downregulation of $\mathrm{miR}-34 \mathrm{a} / \mathrm{b}$ expression upon infection could together account for the observed phenotypic changes in colon organoids.

\section{Discussion}

We demonstrate here the transformation of normal, healthy primary epithelial cells to a premalignant state upon short-term exposure in vitro to a bacterium that is a frequent constituent of the human intestinal microbiota. Specifically, infection of colon organoid cells with colibactin-producing $p k s+E$. coli led to the generation of mutant organoids that grew independently of Wnt. Aberrant Wnt signaling is observed in over $94 \%$ of CRC cases ${ }^{4}$. Also, niche factors have been shown to become dispensable for cancer organoids ${ }^{2,3}$. By selecting for Wnt-independence, we enriched for organoids that were mutated after exposure to $p k s+$ E. coli out of a pool of non-affected or differently mutated cells. Importantly, to capture these clones, short-term infection with colibactin-producing E. coli was sufficient, and no Wntindependent organoids were generated by infecting with an isogenic mutant defective for colibactin synthesis. This observation suggests a 'hit-and-run' mechanism for the transformation of normal primary cells to Wnt-independence upon contact with pks+ E. coli.

The Wnt-independent organoids generated upon infection exhibited accelerated growth, increased organoid formation, and prolonged expandability in culture. Additional signs of transformation included increased expression of Wnt target genes, loss of differentiation markers, and metabolic resemblance of these organoids to undifferentiated cells. Our extensive sequence analysis of Wnt-independent organoids generated by independently infected colon organoid lines revealed a plethora of mutations but none of them directly affected classical Wnt-signaling genes, such as $A p c$, which is often mutated in human colon cancer ${ }^{4}$. Nevertheless, the Wnt pathway was mostly affected indirectly by other mutational changes, e.g., in regulatory genes also frequently seen in human CRC. Likewise, $m i R-34$ members that exhibit defects in a subset of human cancers were heterozygously deleted and found to be downregulated in both replicates. miR-34a is known to be activated by $\mathrm{p} 53$ and downregulates Wnt signaling ${ }^{39}$. miR-34a was found to be silenced in approximately $75 \%$ and $\mathrm{miR}-34 \mathrm{~b} / \mathrm{c}$ in approximately $99 \%$ of sporadic CRC tumor samples ${ }^{43,44}$. In $A p c^{\mathrm{Min} /+}$ mice, miR-34a/b/c have been shown to suppress adenoma formation ${ }^{45}$. Ectopic expression of miR-34 induces cell cycle arrest in primary and tumor-derived cell lines ${ }^{46}$. miR-34a is also known to contribute to p53-mediated apoptosis and EMT suppression $^{42,47}$. Moreover, $m i R-34 a$ loss suppresses asymmetric division and increases $\mathrm{Lgr}^{+}$intestinal stem cell proliferation under inflammatory stress ${ }^{48,49}$. Interestingly, our infectiontransformed organoids exhibited increased nuclear localization of p53 and resistance to Nutlin-3a. Heterozygouos loss of miR34- $a$ and downregulation of $\mathrm{miR}-34 \mathrm{a} / \mathrm{b}$ expression in the Wntindependent organoids could potentially contribute to Nutlin-3a resistance. Further, our results showed that $\operatorname{Trp} 53 \mathrm{KO}$ and $m i R$ $34 a / b / c$ KO colon organoids can grow Wnt-independently, in 
a
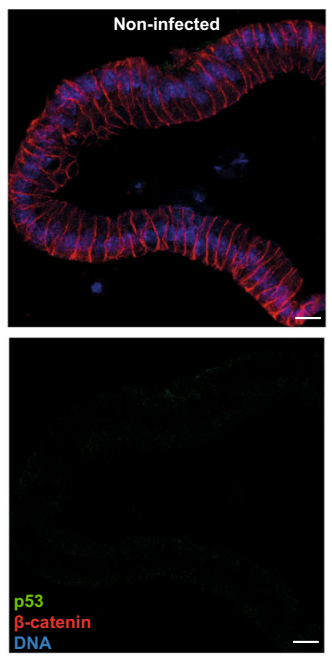

C

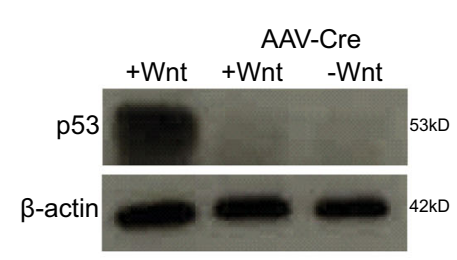

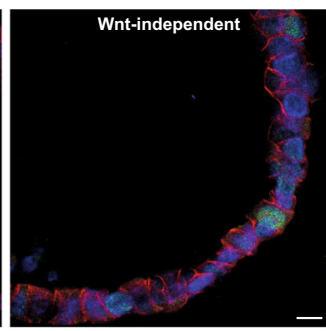

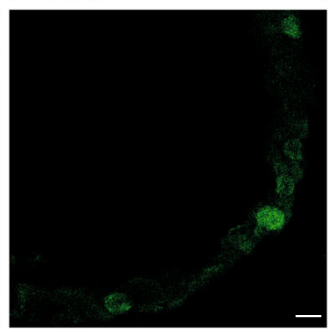

d
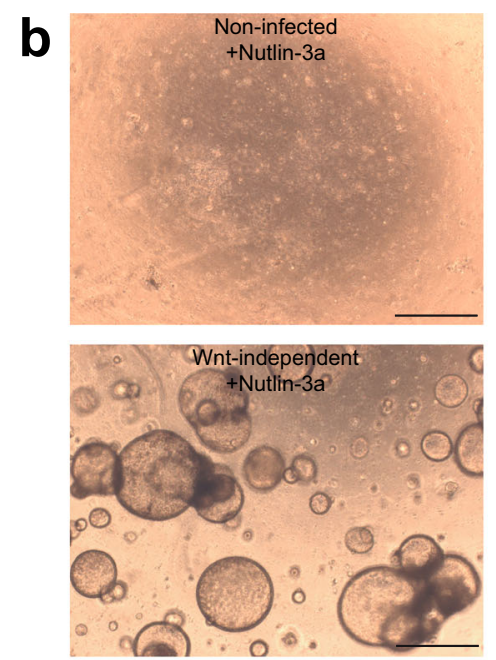

Organoids from Trp53 $5^{\text {foxflox }}$ mice
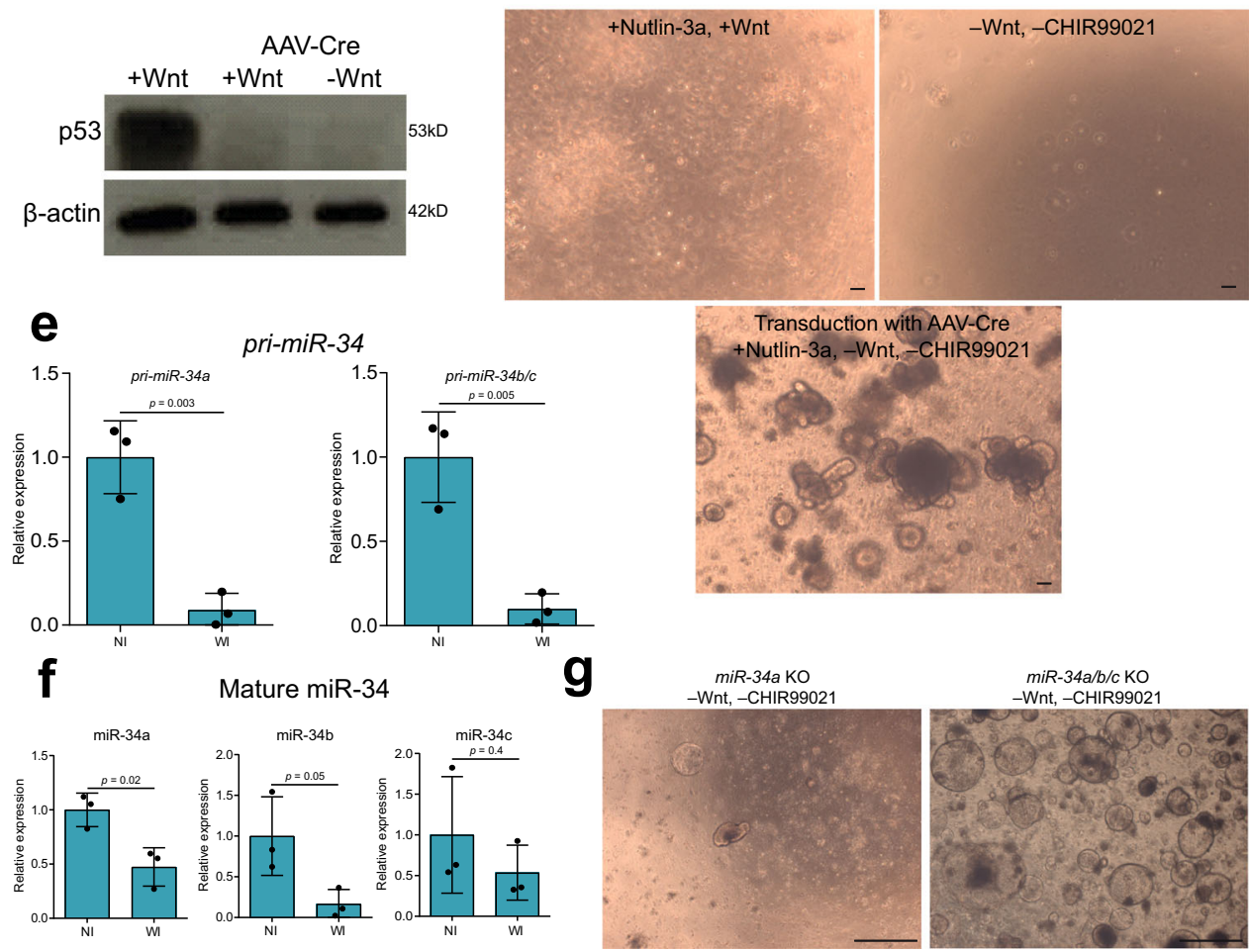

Fig. 5 The Trp53/miR-34 axis is disrupted in the Wnt-independent organoids. a Organoids immunostained for p53 (green) and $\beta$-catenin (red). DAPI (blue) was used to stain for DNA. Image representative of three independent replicates. Scale bars: $10 \mu \mathrm{m}$. b Wnt-independent organoids grow in the presence of Nutlin-3a in the medium. Image representative of three independent replicates. Scale bars: $1 \mathrm{~mm}$. c Western blot to verify knockout of Trp53 in organoids after transduction with AAV-Cre. Image representative of two independent replicates. d Organoids from Trp53flox/flox mice grow in the presence of Nutlin-3a and absence of Wnt and CHIR99021 only when transduced with AAV-Cre. Image representative of two independent replicates. Scale bars: $100 \mu \mathrm{m}$. e RT-qPCR data showing expression of pri-miR-34 relative to TUBB from three independent replicates. Data represent mean \pm SD, $p<0.05$, as calculated by two-sided unpaired Student's $t$-test. $\mathrm{NI}=$ non-infected and $\mathrm{WI}=$ Wnt-independent. $\mathbf{f} \mathrm{RT}$-qPCR data showing expression of mature miR-34 relative to U6 from three independent replicates. Data represent mean $\pm \mathrm{SD}, p<0.05$, as calculated by two-sided unpaired Student's $t$-test. $\mathrm{NI}=$ noninfected and $\mathrm{WI}=$ Wnt-independent. $\mathbf{g}$ Organoids from miR-34a KO mice do not grow in the absence of Wnt and CHIR99021, whereas organoids from $\mathrm{miR}-34 \mathrm{a} / \mathrm{b} / \mathrm{c}$ KO mice do grow in the absence of Wnt and CHIR99021. Image representative of two independent replicates. Scale bars: $1 \mathrm{~mm}$. Source data are provided as a Source Data file.

concordance with previous work showing a functional overlap between these pathways ${ }^{39}$. TP53 mutations are known to occur early in a subset of CRC, such as colitis-associated cancer ${ }^{7}$. Given the diversity of mutational patterns conferring Wnt-independence, considered as the hallmark of colon cancer initiation, it seems plausible that mutations other than the APC mutations frequently seen in advanced stages of cancer may arise at very early stages in the sequence of transforming events, yet have escaped attention. Mutations affecting $m i R-34 a$, as generated here by pks+ E. coli, may be such an example. Therefore, our data strongly suggest that disruption of the p53/miR-34 axis, along with additional mutations that occurred synergistically, resulted in the 
Wnt-independent phenotype in colon organoids. Such mutant cells displaying increased pro-survival signaling may possess a competitive growth advantage over normal cells and grow out to premalignant lesions, including polyps, in affected patients.

The mutational changes in the Wnt-independent organoids included SNV, but mainly CNV. Surprisingly, in these short-term infected organoids we failed to identify the specific mutational signature that we and others have recently shown to be induced by colibactin ${ }^{23,24}$. Notably, these previous studies derived from different experimental settings that aimed for the induction of high DNA damage or mutational burdens, yet did not explore the transforming potential of colibactin. In contrast, here, we exposed primary colon cells to $p k s+E$. coli only for very short times, reminiscent of the conditions in the dynamic process of crypt regeneration in the colon. This short exposure to colibactin sufficed to transform cells, suggesting that mutational changes distinct from the accretion of the recently published colibactin signature were involved.

Obviously, the characteristic colibactin signature is the result of an intricate damage and repair process ensuring minimal localized damage. Any failures to properly resolve the colibactin-induced cross-links, however, would lead to rather crude types of damage, such as severe chromosomal aberrations, in case mitosis proceed $s^{50}$. This could explain the occurrence of multinucleated cells and aneuploidy as observed here, consistent with the occurrence of CIN, previously seen in intestinal epithelial cells after exposure to $p k s+E$. colit ${ }^{16}$. Resulting CNV have been identified as a predominant characteristic of the majority $(\sim 85 \%)$ of sporadic $\mathrm{CRCs}^{51}$, and are observed at early stages of the malignancy, facilitating cancer progression ${ }^{52}$. While in most cases mitotic stress due to aberrant or damaged chromosomes leads to mitotic arrest or cell death, some cells might evade this fate by mitotic slippage ${ }^{53}$. Accordingly, the occurrence of CIN and CNV following colibactin treatment is thought to be the result of inefficient local resolution of cross-links at the damaged sites, rendering cells prone to transformation, e.g., Wnt-independent growth.

The previous identification of the colibactin mutational signature $^{23,24}$ in colon cancer genomes established a clear link between colibactin and the etiology of certain CRCs. The lessdefined chromosomal aberrations resulting from improper repair of colibactin-induced damage, however, might play a prevailing role in cell transformation. Accordingly, the cancer-inducing potential of colibactin-producing bacteria might be higher than the frequency of the colibactin mutational signature suggests. Notably, the specific colibactin signature is found only in $2.4-10 \%$ of patients with $\mathrm{CRC}^{23,24}$, while colibactin-producing E. coli are found in $67 \%$ of CRC patients ${ }^{17}$.

We also observed variability in the genotoxicity between commensal E. coli strains. E. coli M1/5 is slightly more genotoxic than E. coli Nissle 1917, which is commonly used as a probiotic ${ }^{54}$. While we demonstrate that E. coli Nissle 1917 also causes DSBs and cross-links DNA, its damaging activity appears to be below the threshold, incapable of transforming cells to Wntindependence in our assay. However, based on results in vitro, it is difficult to estimate the exact relative genotoxic activity in vivo, since it is subject to regulation by various factors, most prominently the availability of iron in the microenvironment of the infection at the pathogen-host cell interface ${ }^{55,56}$. While additional and as yet uncertain modulatory factors of colibactin expression, production, and delivery may play a role in terms of DNA damage-induction and cancer promotion, at this point it would appear prudent to view the use of colibactin-producing bacteria as probiotics with caution.

Taken together, our data demonstrate the transforming potential of pks + E. coli in vitro and recapitulate the early stages of malignant transformation through infection of colon organoids. In particular, it highlights the relevance of CNV mutations leading to Wnt-independence as early drivers in colon cancer. While the colibactin mutational signature provides a clear link between colibactin action and colon carcinogenesis, colibactin's transforming activity is likely higher than what can be estimated from the occurrence of that signature.

\section{Methods}

Bacterial strains, cell line, and mouse strains. The WT E. coli B2 pks+ M 1/5 human fecal isolate strain was used for the infections. The isogenic mutant strain used has $c l b R$ gene deleted in the $p k s$ island, which substantially hinders the ability of the bacterium to synthesize colibactin ${ }^{26}$. The WT and the mutant E. coli $\Delta c l b R$ strain have a rpsL K42R mutation, which renders the strain streptomycin-resistant. For Supplementary Fig. 4, E. coli Nissle 1917 and E. coli Nissle $1917 \Delta \mathrm{clbB}$ strains were used. The cell line used was human colorectal adenocarcinoma cells Caco-2 (ATCC HTB-37). The cells were cultured in Dulbecco's Modified Eagle Medium (DMEM, Gibco) $+20 \%$ fetal calf serum (FCS, Biochrom). The mouse strains used were mTmG (JAX stock \#007676) ${ }^{57}$, Trp53 flox/flox $(\text { Jackson stock \#008462) })^{58}, \mathrm{miR}$ $34 a \mathrm{KO}^{45}, m i R-34 b / c \mathrm{KO}^{45}$, and $m i R-34 a / b / c \mathrm{KO}^{45}$. All procedures involving animals were approved by the institutional and legal authorities at the Max Planck Institute for Infection Biology (Landesamt für Gesundheit und Soziales, Berlin). All animals were maintained in autoclaved microisolator cages and provided with sterile drinking water and chow ad libitum. Male 4-12 week old mice were used for this study.

Generation of mutant $\boldsymbol{E}$. coli $\Delta \mathbf{c l b R}$. The deletion of $c l b R$ in $E$. coli strain M1/5 has been generated by lambda red-mediated recombineering ${ }^{59}$. A npt kanamycin resistance cassette was amplified from pKD4 (with primer pair GATAAGTTCAAA GAAAAAAACCCGTTATCTCTGCGTGAAAGACAAGTATTGCGCATG CTGTGTAGGCTGGAGCTGCTTC / ATATGAAAATCAATATTATC GACGGCTCAGAAGTGTCTAGATTATCCGTGGCGAT CATATGAATAT CCTCCTTAGTTCC) and used for replacement of the clbRA genes in E. coli strain M1/5 (pKD46). The resulting mutant E. coli M1/5 $\Delta c l b R A$ (pKD46) was then transformed with a PCR product amplified from pKD3- $\triangle c l b R 1$ (primer pair ATATGAAAATCAATATTATCGACGGCTCAGAAGTGTCTAGATTA TCCGTGGCGATCATATGAATATCCTCCTTAGTTCC/ACCGTTGTATCGTA AATTCCTC). The PCR product included $132 \mathrm{bp}$ of the $c l b R$ upstream region, the complete $c l b A$ gene, a cat cassette, an FRT site, and 55 nucleotides of the $c l b A$ downstream sequence. The PCR product was chromosomally inserted via recombination of two FRT site sequences upstream of $n p t$ and downstream of cat. The chromosomal $n p t$ and cat cassettes were then removed by transformation of this strain with pCP20 encoding the FLP recombinase ${ }^{60}$.

Murine colon organoid culture, passaging, and cloning. The mice were sacrificed, and the colons isolated. The colons were cut longitudinally and cleaned with 1X phosphate-buffered saline (PBS) (Gibco), cut into small pieces, and the tissue fragments incubated in a cold chelating buffer (distilled water with $2 \mathrm{mM}$ EDTA, $5.6 \mathrm{mM} \mathrm{Na}_{2} \mathrm{HPO}_{4}, 8 \mathrm{mM} \mathrm{KH}_{2} \mathrm{PO}_{4}, 96.2 \mathrm{mM} \mathrm{NaCl}, 1.6 \mathrm{mM} \mathrm{KCl}, 43.4 \mathrm{mM}$ sucrose, $54.9 \mathrm{mM}$ D-sorbitol, $0.5 \mathrm{mM} \mathrm{DL}$-dithiothreitol) for $30 \mathrm{~min}$ at $4{ }^{\circ} \mathrm{C}$ on a roller mixer. The tube was vigorously shaken to resuspend tissue fragments and to isolate the colon crypts. The tissue fragments were allowed to settle by gravity, and the supernatant containing the crypts was removed and centrifuged at $300 \mathrm{~g}$ for $5 \mathrm{~min}$ at $4{ }^{\circ} \mathrm{C}$. The pellet was resuspended in Advanced DMEM/F-12 (Gibco) containing $10 \%$ heat-inactivated FCS and centrifuged at $300 \mathrm{~g}$ for $5 \mathrm{~min}$ at $4{ }^{\circ} \mathrm{C}$. The pellet was resuspended in $1 \mathrm{ml}$ Advanced DMEM/F-12 containing 10\% FCS and the number of crypts were counted using a hemocytometer. A total of 500 crypts were resuspended and seeded in a $40 \mu \mathrm{l}$ Matrigel (Corning) drop in a 24 -well plate. The Matrigel was polymerized at $37^{\circ} \mathrm{C}$ for $10 \mathrm{~min}$ and then supplemented with $500 \mu \mathrm{l}$ medium: Advanced DMEM/F-12 supplemented with $10 \mathrm{mM}$ HEPES (Gibco), 1\% Glutamax (Gibco), 50\% Wnt-3A conditioned medium, 25\% R-spondin-1 conditioned medium, $100 \mathrm{U} / \mathrm{ml}$ penicillin/streptomycin (Gibco), 1X N2 (Gibco), 1X B27 (Gibco), $1.25 \mathrm{mM}$ N-acetylcysteine (Sigma), $50 \mathrm{ng} / \mathrm{ml}$ murine epidermal growth factor (mEGF) (Invitrogen), $100 \mathrm{ng} / \mathrm{ml}$ murine noggin (Peprotech), $0.5 \mu \mathrm{M} \mathrm{A}-83$ 01 (Sigma), $3 \mu \mathrm{M}$ CHIR99021 (Stemgent), and $9 \mu \mathrm{M}$ Y-27632 dihydrochloride (Sigma). The organoids were incubated at $37^{\circ} \mathrm{C}, 5 \% \mathrm{CO}_{2}$ in a humidified incubator. To the medium, $10 \mu \mathrm{g} / \mathrm{ml}$ metronidazole (Sigma) and $10 \mu \mathrm{g} / \mathrm{ml}$ ciprofloxacin (Bayer) were added additionally for the first 3 days. The medium was changed twice a week. Every 7 days, the organoids were passaged 1:2-1:5 by mechanically disrupting organoids and Matrigel with cold $1 \mathrm{X}$ PBS before transferring into a 15 $\mathrm{ml}$ falcon tube. After centrifugation at $300 \mathrm{~g}$ for $5 \mathrm{~min}$ at $4{ }^{\circ} \mathrm{C}$, the pellet was treated with TrypLE (Gibco) and incubated for $5 \mathrm{~min}$ at $37^{\circ} \mathrm{C}$, followed by dissociation using a flame-polished glass Pasteur pipette. The dissociated organoids were washed with $10 \mathrm{ml}$ Advanced DMEM/F-12 containing 10\% FCS and centrifuged at $300 \mathrm{~g}$ for $5 \mathrm{~min}$ at $4{ }^{\circ} \mathrm{C}$. The pellet was resuspended in $1 \mathrm{ml}$ advanced DMEM/F-12 containing $10 \%$ FCS, and the number of cells counted using a hemocytometer. The required number of cells were resuspended and seeded per $40 \mu$ l Matrigel drop in a 24-well plate. The Matrigel was polymerized for $10 \mathrm{~min}$ at $37^{\circ} \mathrm{C}$. Each well was supplemented with $500 \mu \mathrm{l}$ of medium and incubated at $37^{\circ} \mathrm{C}, 5 \% \mathrm{CO}_{2}$ in a 
humidified incubator. For generating clones, a single organoid was isolated and passaged to obtain the required number of cells. The concentration of cisplatin (Sigma) was $50 \mu \mathrm{M}$ for monolayers and $10 \mu \mathrm{M}$ for organoids. Etoposide (Sigma) was used at $50 \mu \mathrm{M}$ concentration, IWP-2 (Sigma) was used at $5 \mu \mathrm{M}$ concentration, and Nutlin-3a (Sigma) was used at $10 \mu \mathrm{M}$ concentration.

Human colon organoid culture. The human colon biopsies and tissue samples were received from surgeries performed at the Charité University Medicine, with prior approval of the ethics committee of the Charité University Medicine, Berlin (EA1/300/15) and informed consent was obtained from all donors. The biopsies were kept in ChillProtec medium (Biochrom) at $4{ }^{\circ} \mathrm{C}$, and the isolation of crypts for organoid culture was carried out within $2-3 \mathrm{~h}$ after surgery. The tissue was washed with $1 \mathrm{X}$ PBS and cut into small pieces, and the tissue fragments were incubated in a $1 \mathrm{X}$ cold chelating buffer for $30 \mathrm{~min}$ at $4^{\circ} \mathrm{C}$ on a roller mixer. The remaining steps were the same as for the murine colon organoid culture. The human colon organoid medium contained Advanced DMEM/F-12 supplemented with $10 \mathrm{mM}$ HEPES (Gibco), $1 \%$ Glutamax (Gibco), $38 \mathrm{ng} / \mathrm{ml}$ surrogate Wnt (U-Protein Express BV), 25\% R-spondin-1 conditioned medium, $100 \mathrm{U} / \mathrm{ml}$ penicillin/streptomycin (Gibco), 1X N2 (Gibco), 1X B27 (Gibco), $1.25 \mathrm{mM} \mathrm{N}$-acetylcysteine (Sigma), $10 \mathrm{ng} / \mathrm{ml}$ human epidermal growth factor (hEGF) (Invitrogen), $100 \mathrm{ng} / \mathrm{ml}$ human noggin (Peprotech), $0.5 \mu \mathrm{M} \mathrm{A-83-01} \mathrm{(Sigma),} 10 \mathrm{mM}$ nicotinamide (Sigma), $10 \mu \mathrm{M}$ SB202190 (Sigma), $10 \mathrm{nM}$ prostaglandin E2 (Tocris), and $9 \mu \mathrm{M} \mathrm{Y}$ 27632 dihydrochloride (Sigma). The organoids were incubated at $37^{\circ} \mathrm{C}, 5 \% \mathrm{CO}_{2}$ in a humidified incubator. For the first 3 days, $100 \mu \mathrm{g} / \mathrm{ml}$ Primocin (Invivogen) was additionally added to the medium. The medium was changed twice a week. The passaging protocol was the same as for the murine colon organoids.

Murine primary colon epithelial monolayer culture. Half a million cells derived from murine colon organoids were resuspended in $200 \mu \mathrm{l}$ of medium and seeded into bovine collagen type I-coated (Gibco, $1 \mathrm{mg} / \mathrm{ml}$ ) polycarbonate cell culture inserts (Merck Millicell) placed in a 24-well plate. For monolayers, the medium used for organoids was supplemented with $10 \%$ FCS. Outside the cell culture insert, $600 \mu \mathrm{l}$ medium was added, and the plate was placed at $37^{\circ} \mathrm{C}, 5 \% \mathrm{CO}_{2}$ in a humidified incubator. After 3 days, the medium from the cell culture inserts was discarded. The medium outside the cell culture insert was changed twice each week.

In vitro infections. We determined that MOI of 20 and MOI of 5 was ideal for Caco-2 and primary cells, respectively, to obtain DNA damage after a $3 \mathrm{~h}$ infection. Higher MOIs caused very high or absolute cell death. Caco-2 cells were grown on collagen-coated MatTek glass-bottom dishes. For infection of Caco-2 cells, the medium was removed from $70 \%$ confluent cells. Infection medium (DMEM containing $10 \%$ FCS and $10 \mathrm{mM}$ HEPES) containing log-phase E. coli at MOI 20 was added to the cells. The cells were then kept for $3 \mathrm{~h}$ in an incubator at $37^{\circ} \mathrm{C}$. To stop the infection, cell culture medium containing $200 \mu \mathrm{g} / \mathrm{ml}$ gentamicin (Sigma) was added, and cells were incubated at $37^{\circ} \mathrm{C}$ overnight. The next day, the medium was changed to medium without gentamicin.

For infection of organoids, Matrigel was mechanically disrupted with infection medium and transferred to a $15 \mathrm{ml}$ Falcon tube. The organoids were broken up by passing them through a flame-polished glass Pasteur pipette, so that both the basal and apical sides were exposed to the bacteria. The organoids were centrifuged at $300 \mathrm{~g}$ for $5 \mathrm{~min}$ at $4{ }^{\circ} \mathrm{C}$ in a $1.5 \mathrm{ml}$ tube and resuspended in $250 \mu \mathrm{l}$ of infection medium. Log-phase E. coli at MOI 5 were added, and the tube incubated for $3 \mathrm{~h}$ at $37^{\circ} \mathrm{C}$. The organoids were centrifuged at $300 \mathrm{~g}$ for $5 \mathrm{~min}$ at $4{ }^{\circ} \mathrm{C}$ and washed 2 times with advanced DMEM/F-12 containing $10 \%$ FCS. The pellet was resuspended in the required amount of Matrigel, and $40 \mu \mathrm{l}$ drops added to each well in a 24-well plate. The Matrigel was polymerized by placing at $37^{\circ} \mathrm{C}$ for $10 \mathrm{~min}$. Each well was supplemented with $500 \mu \mathrm{l}$ of medium containing $200 \mu \mathrm{g} / \mathrm{ml}$ gentamicin. The plate was then incubated at $37^{\circ} \mathrm{C}$. The next day, the medium was changed to medium without gentamicin.

Monolayers were infected by adding $250 \mu \mathrm{l}$ of infection medium containing logphase E. coli at MOI 5 to the cell culture inserts and incubating at $37^{\circ} \mathrm{C}$ for $3 \mathrm{~h}$.

For obtaining CFUs, after $3 \mathrm{~h}$ of infection, the organoids were washed with $1 \mathrm{X}$ PBS 5 times. The organoids were then treated with $0.2 \%$ saponin (Sigma) for 10 min at $37^{\circ} \mathrm{C}$ to dissociate the attached bacteria. Serial dilutions were made and plated on LB agar plates, and colonies were counted the following day.

Immunofluorescence. Caco-2 cells were grown on MatTek glass-bottom dishes and washed with PBS and fixed with $3.7 \%$ paraformaldehyde (Sigma) for $1 \mathrm{~h}$. Organoids were mechanically disrupted and dissociated from Matrigel with cold PBS, transferred into a glass tube and allowed to settle by gravity. The organoids were then fixed with $3.7 \%$ paraformaldehyde for $1 \mathrm{~h}$. Monolayers were washed with PBS and fixed in $3.7 \%$ paraformaldehyde for $1 \mathrm{~h}$. They were first embedded in histogel (Thermo Scientific), followed by embedding in paraffin (Carl Roth). After fixation, the tissue pieces, organoids, and monolayers were dehydrated $(1 \mathrm{~h} \mathrm{incu-}$ bations in $70 \% \mathrm{EtOH}, 80 \% \mathrm{EtOH}, 90 \% \mathrm{EtOH}$, absolute $\mathrm{EtOH}, 100 \%$ isopropanol, twice in xylene) and paraffinized, cut into $5 \mu \mathrm{m}$ sections with a microtome, and mounted on glass slides before dewaxing. For whole-mount staining of organoids, they were fixed directly in the Matrigel by adding $500 \mu \mathrm{l}$ of 3.7\% paraformaldehyde for half an hour at room temperature, then washed with 1X PBS before labeling.
For whole-mount staining of monolayers, the monolayers were washed with PBS and fixed in 3.7\% paraformaldehyde for $1 \mathrm{~h}$, and then the filter was cut out and stained. The staining was performed with the antibodies and dyes listed in Supplementary Table 1. Primary antibodies were diluted in blocking solution (1\% BSA, $2 \%$ FCS, and $0.1 \%$ Tween 20 in 1X PBS) and incubated overnight at $4{ }^{\circ} \mathrm{C}$. Secondary antibodies were diluted in blocking solution, incubated for $45 \mathrm{~min}$ at room temperature for paraffin sections, and overnight at $4{ }^{\circ} \mathrm{C}$ for whole-mount. Mounting was done with Mowiol or Vectashield mounting medium (Vectorlabs, Inc.). Images were acquired with a Leica TCS SP-8 confocal microscope and processed using ImageJ 1.52 .

Western Immunoblotting. For the western blots, organoids were harvested in $2 \mathrm{X}$ Laemmli buffer, separated by 10\% SDS-PAGE gels, transferred to a PVDF membrane (PerkinElmer), blocked in TBS buffer supplemented with $0.1 \%$ Tween 20 (Merck) and 5\% milk (AppliChem), and probed against primary antibodies p53 DO-1 (Santa Cruz Biotechnology, SC-126, 1:500 dilution) and $\beta$-actin (SigmaAldrich, A5441, 1:10000 dilution) at $4{ }^{\circ} \mathrm{C}$ overnight. Membranes were probed with matching secondary horseradish-peroxidase-conjugated antibodies (Amersham, 1:3,000) and detected with ECL reagent (PerkinElmer). An uncropped scan image of the blot is provided in the Source Data file.

Real-Time qPCR. Organoids were released from Matrigel with cold 1X PBS and pelleted by centrifugation $\left(300 \mathrm{~g}\right.$ for $5 \mathrm{~min}$ at $4{ }^{\circ} \mathrm{C}$ ), followed by RNA isolation using the phenol-chloroform RNA extraction method. For primary miRNA, total RNA was isolated using the miRNeasy mini kit (Qiagen), and for analysis, $1 \mu \mathrm{g}$ of total RNA per sample was used to generate CDNA using the Verso cDNA synthesis kit (Thermo Fisher). qPCR was performed using a Power SYBR Green RNA-to-CT 1-Step Kit (Applied Biosystems). Reactions were performed in $25 \mu \mathrm{l}$ containing 50-200 ng RNA, $10 \mu \mathrm{l}$ SYBR Green mix, $0.16 \mu \mathrm{l}$ RT mix, plus primers at $0.2 \mu \mathrm{M}$ each. Program: $30 \mathrm{~min}$ at $48^{\circ} \mathrm{C} ; 10 \mathrm{~min}$ at $95^{\circ} \mathrm{C}$; followed by 40 cycles of $15 \mathrm{~s}$ at $95^{\circ} \mathrm{C} / 60 \mathrm{~s}$ at $60^{\circ} \mathrm{C}$. For analyses of mature miRNA expression, cDNA was generated, and $\mathrm{qPCR}$ was performed using the miRCURY LNA RT kit (Qiagen), the miRCURY LNA $A^{\text {st }}$ SYBR Green Master Mix (Exiqon), and the following commercially available primers: mmu-miR-34a (Qiagen, YP00204486), mmu-miR-34b (Qiagen, YP00204424), mmu-miR-34c (Qiagen, YP00205659), and the snRNA U6 (Exiqon, 203907). Primers used are listed in Supplementary Table 2. For each oligonucleotide pair and RNA sample, the reaction was performed in triplicate. The data was analyzed using the delta-Cq method. The expression levels of the target genes were normalized to the levels of glyceraldehyde-3-phosphate dehydrogenase (Gapdh) gene expression in each individual sample. For primary miRNA, the expression was normalized to TUBB, and for miRNA, the expression was normalized to U6.

DNA cross-linking assay. This method was adapted from Xue et al. ${ }^{61}$. Here, $1 \mathrm{ml}$ of overnight culture was centrifuged at $1600 \mathrm{~g}$ for $3 \mathrm{~min}$. The pellet was resuspended in $20 \mathrm{ml} \mathrm{M9-CAP} \mathrm{medium} \mathrm{and} \mathrm{shaken} \mathrm{at} 200 \mathrm{rpm}$ for $2 \mathrm{~h}$ at $37^{\circ} \mathrm{C}$. M9-CAP medium contained M9 minimum media (Difco) supplemented with $0.4 \%$ glucose, $2 \mathrm{mM} \mathrm{MgSO}_{4}, 0.1 \mathrm{mM} \mathrm{CaCl}_{2}, 12.5 \mu \mathrm{g} / \mathrm{ml}$ chloramphenicol, and the following Lamino acid mass composition ( $5 \mathrm{~g} / \mathrm{l}$ total): $3.6 \%$ Arg, $21.1 \%$ Glu, $2.7 \%$ His, $5.6 \%$ Ile, 8.4\% Leu, 7.5\% Lys, $4.6 \%$ Phe, $4.2 \%$ Thr, 1.1\% Trp, 6.1\% Tyr, 5\% Val, $4 \%$ Asn, $4 \%$ Ala, $4 \%$ Met, $4 \%$ Gly, $4 \%$ Cys, and $4 \%$ Ser. At this time point, OD measured at $600 \mathrm{~nm}$ is 0.8 and there are $1 \times 10^{9} \mathrm{CFUs} / \mathrm{ml}$. Serially diluted bacteria were added to $1.5 \mu \mathrm{g}$ of pfdA8 plasmid DNA containing $3 \mu \mathrm{l}$ of $50 \mathrm{mM}$ EDTA and incubated for $90 \mathrm{~min}$ at $37^{\circ} \mathrm{C}$. Cleanup of DNA was done using QIAquick PCR Purification Kit (Qiagen), and $300 \mathrm{ng}$ of DNA samples were loaded in a $1 \%$ denaturing agarose gel ( $1 \%$ agarose, $5 \% 1 \mathrm{M} \mathrm{NaCl}$, and $2 \% 50 \mathrm{mM}$ EDTA water). The gel ran at $50 \mathrm{~V}$ for $2 \mathrm{~h}$ in running buffer $(5 \% 1 \mathrm{M} \mathrm{NaOH}$ and $0.2 \% 0.5 \mathrm{M}$ EDTA in water). The DNA was stained with SafeRed (Biotium), and the gel was imaged under UV light.

Neutral comet assay. The neutral comet assay was performed following infection, etoposide (Sigma) treatment or cisplatin (Sigma) treatment of single cells derived from organoids using the kit from Trevigen $(4250-050-\mathrm{K})$, following the manufacturer's protocol. Images were acquired using a fluorescent microscope (Leica DMR). The percentage of DNA in the tail was quantified using the software CometScore (TriTek 2.0).

Transduction. The organoids were dissociated with TrypLE and resuspended in $300 \mu \mathrm{l}$ culture medium (without penicillin/streptomycin and N-acetylcysteine) containing AAV-Cre adenovirus particles and $8 \mu \mathrm{g} / \mathrm{ml}$ polybrene (Sigma). Cells were seeded in a 24-well plate coated with Matrigel. After overnight incubation, the medium was removed, and a second layer of Matrigel was added and polymerized as described above, before adding $500 \mu \mathrm{l}$ culture medium. The culture was passaged after 7 days.

RNA sequencing. RNA sequencing was performed at the Max Planck Genome Centre, Cologne, using Illumina HiSeq3000. PolyA enrichment was applied to all samples. For each sample of replicate 1 , about $50 \mathrm{M}$ single-end reads $(150 \mathrm{bp})$ were generated. For replicates 2 and 3, about $20 \mathrm{M}$ paired-end reads $(2 \times 150 \mathrm{bp})$ were generated per sample. Gene expression was quantified by Salmon ${ }^{62}$, using pseudo- 
alignment of unaligned reads against transcript sequences from Gencode M12 (www.gencode.org). Gene quantification data generated by Salmon were further processed in R using package DESeq $2^{63}$ to determine differentially expressed genes between conditions and samples.

DNA sequencing. The sequencing of non-infected and Wnt-independent organoids for both replicates was performed at passage 11 and passage 13 after infection, respectively. All conditions were compared to all the non-infected organoids of that respective replicate. For each condition, DNA was extracted from approximately 1 million cells.

The exome sequencing was performed at the Max Planck Genome Centre, Cologne, using Illumina HiSeq3000. Exome targets were captured using SureSelectXT Mouse All Exon kit (Agilent Technologies) and sequenced using a paired-end library protocol $(2 \times 150 \mathrm{bp})$ with a target coverage of 60 -fold at $80 \%$ on-target rate. Reads were aligned against mouse genome version GRCm38 using BWA. Duplicate marking, base call recalibration, and realignment around indels were done using GATK v3.4 ${ }^{64}$. Somatic SNPs and small indels were called against pooled non-infected conditions as germline control using Strelka v1.0.11 ${ }^{65}$ and annotated using SnpEff v4.3k ${ }^{66}$. CNV were determined using CONTRA v2.08 ${ }^{67}$

WGS was performed at BGI Tech Solutions, Hong Kong, using BGISEQ-500 with a paired-end $(2 \times 100 \mathrm{bp})$ library protocol and a target coverage of 30 -fold. Reads were mapped to GRCm 38 using BWA. CNV for WGS data were called using ControlFreeC v10.8 ${ }^{68}$ and R package DNAcopy. Copy number segments with absolute $\log 2$ ratio larger than 0.15 were called as gains or losses. Structural variants were called using SvABA ${ }^{69}$. SNV and indels were called in a similar way as for exome variants using GATK preprocessing and variant calling by Strelka.

For both exome and whole-genome sequencing, we kept only SNV and indels with allelic proportions of at least $25 \%$ in order to remove subclonal variants arising during expansion after treatment.

All tertiary analysis was done using $\mathrm{R}^{70}$, including package circlize $\mathrm{e}^{71}$.

Gene set enrichment analysis (GSEA). Gene sets were used from MSigDB v6.2 (collections H, C2, C3, C5, C6, and C7) 72,73 after mapping genes from mouse to human genes using the HomoloGene database ${ }^{74}$. Additionally, lists of intestinal stem cell signature genes ${ }^{31}$ and genes upregulated and downregulated during activated $\beta$-catenin signaling ${ }^{32}$ were used. The GSEA $^{75}$ was performed on genes pre-ranked by gene expression-based $\log 2$ fold change between non-infected and Wnt-independent organoids using the fgsea $\mathrm{R}$ package ${ }^{76}$ with 5000 permutations; R v.3.4 was used (https://cran.r-project.org/). For each experiment, $p$ values from all gene sets were jointly adjusted for multiple testing using the method of Benjamini-Hochberg (false discovery rate) ${ }^{77}$.

Statistical analysis. All graphs were prepared using GraphPad Prism version 8 software. Data are presented as mean \pm SD (standard deviation), and $p$ values were calculated by two-sided unpaired Student's $t$-test. For comet assays, one-way ANOVA with respective post hoc analysis (Tukey's test) was used to calculate $p$ values. Data were considered significant if $p<0.05$. Unless stated otherwise, figures represent three independent experiments.

Reporting summary. Further information on research design is available in the Nature Research Reporting Summary linked to this article.

\section{Data availability}

All RNA sequencing data are available from Gene Expression Omnibus (GEO) with ID GSE140929. Whole-genome and exome sequencing data have been submitted to the European Nucleotide Archive (ENA) under accession PRJEB35529. MSigDB v6.2 is available from http://www.gsea-msigdb.org/gsea/downloads_archive.jsp. Homologene build 68 is available from https://ftp.ncbi.nih.gov/pub/HomoloGene/. Gencode M12 mouse gene models were obtained from ftp://ftp.ebi.ac.uk/pub/databases/gencode/ Gencode_mouse/release_M12/. Other data supporting the findings of this study are available within the paper and its Supplementary Information files, or from the corresponding authors upon request. Source data are provided with this paper.

\section{Code availability}

All code is available from https:/github.com/MPIIB-Department-TFMeyer/ Iftekhar_Colibactin. The DOI link is https://doi.org/10.5281/zenodo.432272978.

Received: 9 November 2020; Accepted: 13 January 2021; Published online: 12 February 2021

\section{References}

1. Fearon, E. R. \& Vogelstein, B. A genetic model for colorectal tumorigenesis. Cell 61, 759-767 (1990).

2. Drost, J. et al. Sequential cancer mutations in cultured human intestinal stem cells. Nature 521, 43-47 (2015).
3. Matano, M. et al. Modeling colorectal cancer using CRISPR-Cas9-mediated engineering of human intestinal organoids. Nat. Med. 21, 256-262 (2015).

4. The Cancer Genome Atlas Network Comprehensive molecular characterization of human colon and rectal cancer. Nature 487, 330-337 (2012).

5. Barker, N. et al. Identification of stem cells in small intestine and colon by marker gene Lgr5. Nature 449, 1003-1007 (2007).

6. Miyoshi, Y. et al. Somatic mutations of the APC gene in colorectal tumors: mutation cluster region in the APC gene. Hum. Mol. Genet. 1, 229-233 (1992).

7. West, N. R., McCuaig, S., Franchini, F. \& Powrie, F. Emerging cytokine networks in colorectal cancer. Nat. Rev. Immunol. 15, 615-629 (2015).

8. Brentnall, T. A. et al. Mutations in the 553 gene: an early marker of neoplastic progression in ulcerative colitis. Gastroenterology 107, 369-378 (1994).

9. Fujita, M. et al. Genomic landscape of colitis-associated cancer indicates the impact of chronic inflammation and its stratification by mutations in the Wnt signaling. Oncotarget 9, 969-981 (2018).

10. Hussain, S. P. et al. Increased p53 mutation load in noncancerous colon tissue from ulcerative colitis: a cancer-prone chronic inflammatory disease. Cancer Res. 60, 3333-3337 (2000).

11. Scully, R. The spindle-assembly checkpoint, aneuploidy, and gastrointestinal cancer. N. Engl. J. Med. 363, 2665-2666 (2010)

12. Thomas, A. M. et al. Metagenomic analysis of colorectal cancer datasets identifies cross-cohort microbial diagnostic signatures and a link with choline degradation. Nat. Med. 25, 667-678 (2019).

13. Wirbel, J. et al. Meta-analysis of fecal metagenomes reveals global microbial signatures that are specific for colorectal cancer. Nat. Med. 25, 679-689 (2019).

14. Wong, S. H. et al. Gavage of fecal samples from patients with colorectal cancer promotes intestinal carcinogenesis in germ-free and conventional mice. Gastroenterology 153, 1621-1633.e6 (2017).

15. Nougayrede, J. P. et al. Escherichia coli induces DNA double-strand breaks in eukaryotic cells. Science 313, 848-851 (2006).

16. Cuevas-Ramos, G. et al. Escherichia coli induces DNA damage in vivo and triggers genomic instability in mammalian cells. Proc. Natl Acad. Sci. USA 107, 11537-11542 (2010).

17. Arthur, J. C. et al. Intestinal inflammation targets cancer-inducing activity of the microbiota. Science 338, 120-123 (2012).

18. Dejea, C. M. et al. Patients with familial adenomatous polyposis harbor colonic biofilms containing tumorigenic bacteria. Science 359, 592-597 (2018).

19. Prorok-Hamon, M. et al. Colonic mucosa-associated diffusely adherent afaC+ Escherichia coli expressing lpfA and pks are increased in inflammatory bowel disease and colon cancer. Gut 63, 761-770 (2014).

20. Healy, A. R., Nikolayevskiy, H., Patel, J. R., Crawford, J. M. \& Herzon, S. B. A mechanistic model for colibactin-induced genotoxicity. J. Am. Chem. Soc. 138, 15563-15570 (2016)

21. Wilson, M. R. et al. The human gut bacterial genotoxin colibactin alkylates DNA. Science 363, eaar7785 (2019).

22. Xue, M., Shine, E., Wang, W., Crawford, J. M. \& Herzon, S. B. Characterization of natural colibactin-nucleobase adducts by tandem mass spectrometry and isotopic labeling. Support for DNA alkylation by cyclopropane ring opening. Biochemistry 57, 6391-6394 (2018).

23. Dziubanska-Kusibab, P. J. et al. Colibactin DNA-damage signature indicates mutational impact in colorectal cancer. Nat. Med. 26, 1063-1069 (2020).

24. Pleguezuelos-Manzano, C. et al. Mutational signature in colorectal cancer caused by genotoxic pks(+) E. coli. Nature 580, 269-273 (2020).

25. Cougnoux, A. et al. Bacterial genotoxin colibactin promotes colon tumour growth by inducing a senescence-associated secretory phenotype. Gut 63, 1932-1942 (2014)

26. Wallenstein, A. et al. ClbR is the key transcriptional activator of colibactin gene expression in Escherichia coli. mSphere 5, e00591-00520 (2020).

27. Boccellato, F. et al. Polarised epithelial monolayers of the gastric mucosa reveal insights into mucosal homeostasis and defence against infection. Gut 68, 400-413 (2019).

28. Moon, C., VanDussen, K. L., Miyoshi, H. \& Stappenbeck, T. S. Development of a primary mouse intestinal epithelial cell monolayer culture system to evaluate factors that modulate IgA transcytosis. Mucosal Immunol. 7, 818-828 (2014).

29. Sato, T. et al. Long-term expansion of epithelial organoids from human colon, adenoma, adenocarcinoma, and Barrett's epithelium. Gastroenterology 141, 1762-1772 (2011).

30. Takahara, P. M., Rosenzweig, A. C., Frederick, C. A. \& Lippard, S. J. Crystal structure of double-stranded DNA containing the major adduct of the anticancer drug cisplatin. Nature 377, 649-652 (1995).

31. Munoz, J. et al. The Lgr5 intestinal stem cell signature: robust expression of proposed quiescent ' +4 ' cell markers. EMBO J. 31, 3079-3091 (2012).

32. Riemer, P. et al. Oncogenic beta-catenin and PIK3CA instruct network states and cancer phenotypes in intestinal organoids. J. Cell Biol. 216, 1567-1577 (2017). 
33. Sato, T. et al. Paneth cells constitute the niche for Lgr5 stem cells in intestinal crypts. Nature 469, 415-418 (2011).

34. Stephens, P. J. et al. Massive genomic rearrangement acquired in a single catastrophic event during cancer development. Cell 144, 27-40 (2011).

35. Bailey, M. H. et al. Comprehensive characterization of cancer driver genes and mutations. Cell 174, 1034-1035 (2018).

36. Kadoch, C. et al. Proteomic and bioinformatic analysis of mammalian SWI/ SNF complexes identifies extensive roles in human malignancy. Nat. Genet. 45, 592-601 (2013).

37. Mathur, R. et al. ARID1A loss impairs enhancer-mediated gene regulation and drives colon cancer in mice. Nat. Genet. 49, 296-302 (2017).

38. Bitler, B. G. et al. ARID1A-mutated ovarian cancers depend on HDAC6 activity. Nat. Cell Biol. 19, 962-973 (2017).

39. Kim, N. H. et al. p53 and microRNA-34 are suppressors of canonical Wnt signaling. Sci. Signal. 4, ra71 (2011).

40. Rokavec, M., Li, H., Jiang, L. \& Hermeking, H. The p53/miR-34 axis in development and disease. J. Mol. Cell. Biol. 6, 214-230 (2014).

41. Bommer, G. T. et al. p53-mediated activation of miRNA34 candidate tumorsuppressor genes. Curr. Biol. 17, 1298-1307 (2007).

42. Siemens, H. et al. miR-34 and SNAIL form a double-negative feedback loop to regulate epithelial-mesenchymal transitions. Cell Cycle 10, 4256-4271 (2011).

43. Vogt, M. et al. Frequent concomitant inactivation of miR-34a and miR-34b/c by CpG methylation in colorectal, pancreatic, mammary, ovarian, urothelial, and renal cell carcinomas and soft tissue sarcomas. Virchows Arch. 458, 313-322 (2011).

44. Wu, X. D. et al. Detection of miR-34a and miR-34b/c in stool sample as potential screening biomarkers for noninvasive diagnosis of colorectal cancer. Med. Oncol. 31, 894 (2014).

45. Jiang, L. \& Hermeking, H. miR-34a and miR-34b/c suppress intestinal tumorigenesis. Cancer Res. 77, 2746-2758 (2017).

46. He, L. et al. A microRNA component of the $\mathrm{p} 53$ tumour suppressor network. Nature 447, 1130-1134 (2007).

47. Raver-Shapira, N. et al. Transcriptional activation of miR-34a contributes to p53-mediated apoptosis. Mol. Cell 26, 731-743 (2007).

48. $\mathrm{Bu}, \mathrm{P}$. et al. A miR-34a-numb feedforward loop triggered by inflammation regulates asymmetric stem cell division in intestine and colon cancer. Cell Stem Cell 18, 189-202 (2016).

49. Wang, L. et al. miR-34a is a microRNA safeguard for Citrobacter-induced inflammatory colon oncogenesis.eLife 7, e39479 (2018).

50. Gelot, C., Magdalou, I. \& Lopez, B. S. Replication stress in mammalian cells and its consequences for mitosis. Genes (Basel) 6, 267-298 (2015).

51. Grady, W. M. \& Carethers, J. M. Genomic and epigenetic instability in colorectal cancer pathogenesis. Gastroenterology 135, 1079-1099 (2008).

52. Stoler, D. L. et al. The onset and extent of genomic instability in sporadic colorectal tumor progression. Proc. Natl Acad. Sci. USA 96, 15121-15126 (1999).

53. Burgess, A., Rasouli, M. \& Rogers, S. Stressing mitosis to death. Front Oncol. 4, 140 (2014)

54. Olier, M. et al. Genotoxicity of Escherichia coli Nissle 1917 strain cannot be dissociated from its probiotic activity. Gut Microbes 3, 501-509 (2012).

55. Tronnet, S. et al. High iron supply inhibits the synthesis of the genotoxin colibactin by pathogenic Escherichia coli through a non-canonical Fur/RyhBmediated pathway.Pathog. Dis. 75, ftx066 (2017)

56. Tronnet, S. et al. Iron homeostasis regulates the genotoxicity of Escherichia coli that produces colibactin. Infect. Immun. 84, 3358-3368 (2016).

57. Muzumdar, M. D., Tasic, B., Miyamichi, K., Li, L. \& Luo, L. A global doublefluorescent Cre reporter mouse. Genesis 45, 593-605 (2007).

58. Marino, S., Vooijs, M., van Der Gulden, H., Jonkers, J. \& Berns, A. Induction of medulloblastomas in p53-null mutant mice by somatic inactivation of $\mathrm{Rb}$ in the external granular layer cells of the cerebellum. Genes Dev. 14, 994-1004 (2000).

59. Datsenko, K. A. \& Wanner, B. L. One-step inactivation of chromosomal genes in Escherichia coli K-12 using PCR products. Proc. Natl Acad. Sci. USA 97, 6640-6645 (2000).

60. Cherepanov, P. P. \& Wackernagel, W. Gene disruption in Escherichia coli: TcR and $\mathrm{KmR}$ cassettes with the option of Flp-catalyzed excision of the antibioticresistance determinant. Gene 158, 9-14 (1995).

61. Xue, M., Wernke, K. M. \& Herzon, S. B. Depurination of colibactin-derived interstrand cross-links. Biochemistry 59, 892-900 (2020).

62. Patro, R., Duggal, G., Love, M. I., Irizarry, R. A. \& Kingsford, C. Salmon provides fast and bias-aware quantification of transcript expression. Nat. Methods 14, 417-419 (2017).

63. Love, M. I., Huber, W. \& Anders, S. Moderated estimation of fold change and dispersion for RNA-seq data with DESeq2. Genome Biol. 15, 550 (2014).

64. McKenna, A. et al. The Genome Analysis Toolkit: a MapReduce framework for analyzing next-generation DNA sequencing data. Genome Res. 20, 1297-1303 (2010).

65. Saunders, C. T. et al. Strelka: accurate somatic small-variant calling from sequenced tumor-normal sample pairs. Bioinformatics 28, 1811-1817 (2012).
66. Cingolani, P. et al. A program for annotating and predicting the effects of single nucleotide polymorphisms, SnpEff: SNPs in the genome of Drosophila melanogaster strain w1118; iso-2; iso-3. Fly (Austin) 6, 80-92 (2012).

67. Li, J. et al. CONTRA: copy number analysis for targeted resequencing. Bioinformatics 28, 1307-1313 (2012).

68. Boeva, V. et al. Control-FREEC: a tool for assessing copy number and allelic content using next-generation sequencing data. Bioinformatics 28, 423-425 (2012).

69. Wala, J. A. et al. SvABA: genome-wide detection of structural variants and indels by local assembly. Genome Res. 28, 581-591 (2018).

70. R Core Team. R: A Language and Environment for Statistical Computing. https://www.R-project.org/ (R Foundation for Statistical Computing, 2013).

71. Gu, Z., Gu, L., Eils, R., Schlesner, M. \& Brors, B. circlize Implements and enhances circular visualization in R. Bioinformatics 30, 2811-2812 (2014).

72. Liberzon, A. et al. The Molecular Signatures Database (MSigDB) hallmark gene set collection. Cell Syst. 1, 417-425 (2015).

73. Liberzon, A. et al. Molecular signatures database (MSigDB) 3.0. Bioinformatics 27, 1739-1740 (2011)

74. Sayers, E. W. et al. Database resources of the National Center for Biotechnology Information. Nucleic Acids Res. 39, D38-D51 (2011).

75. Subramanian, A. et al. Gene set enrichment analysis: a knowledge-based approach for interpreting genome-wide expression profiles. Proc. Natl Acad. Sci. USA 102, 15545-15550 (2005).

76. Sergushichev, A. A. An algorithm for fast preranked gene set enrichment analysis using cumulative statistic calculation. Preprint at https://www.biorxiv. org/content/10.1101/060012v1 (2016)

77. Benjamini, Y. \& Hochberg, Y. Controlling the false discovery rate: a practical and powerful approach to multiple testing. J. R. Stat. Soc. B 57, 289-300 (1995).

78. Iftekhar, A. et al. Genomic aberrations after short-term exposure to colibactinproducing E. coli transform primary colon epithelial cells. GitHub Repository, https://doi.org/10.5281/zenodo.4322729 (2020).

\section{Acknowledgements}

The authors would like to thank Kirstin Hoffmann, Susan Jackisch, Oliver Thieck, Anja Greiser, and the late Jörg Angermann for excellent technical support; Meike Sörensen for optimizing and performing the cross-linking assay; Dr. Rosa Schmuck and Dr. Rüdiger Schoo for providing human colon samples; Dr. Maria del Mar Reines for sharing her insights into the Wnt and p53 pathways; Dr. Michela Di Virgilio for her advice regarding antibodies for DNA damage repair proteins; Dr. Aki Imai-Matsushima for introducing to us the concept of air-liquid interface culture; Diane Schad for help with generating the graphics; and Dr. Rike Zietlow and Dr. Kfir Lapid for editing the manuscript.

\section{Author contributions}

A.I., M.S., and T.F.M. conceived the study. A.I., H.B., M.S., and T.F.M. designed the experiments. A.I. performed most of the experiments and analyzed the data. M.S. and T.F.M. supervised the study and obtained third party funding. Bioinformatics analyses were performed by H.B.; H.H. provided the miR-34 KO mice and supervised N.B.; N.B generated and analyzed miR-34 KO organoids. J.H. contributed to the cultivation of human colon organoids and performed the $\beta$-catenin staining. F.B. provided critical technical advice. U.D. provided the E. coli strains and respective advice. The manuscript was written by A.I., H.B., M.S., and T.F.M.

\section{Funding}

This work was supported by German Research Foundation (DFG) grants to U.D. (Do789/11-1), M.S. (Sil983 3-1, Si1983 4-1), and T.F.M. (Me705/18-1). Open Access funding enabled and organized by Projekt DEAL.

\section{Competing interests}

The authors declare no competing interests.

\section{Additional information}

Supplementary information The online version contains supplementary material available at https://doi.org/10.1038/s41467-021-21162-y.

Correspondence and requests for materials should be addressed to M.S. or T.F.M

Peer review information Nature Communications thanks the anonymous reviewer(s) for their contribution to the peer review of this work.

Reprints and permission information is available at http://www.nature.com/reprints

Publisher's note Springer Nature remains neutral with regard to jurisdictional claims in published maps and institutional affiliations. 
(c) (i) Open Access This article is licensed under a Creative Commons Attribution 4.0 International License, which permits use, sharing, adaptation, distribution and reproduction in any medium or format, as long as you give appropriate credit to the original author(s) and the source, provide a link to the Creative Commons license, and indicate if changes were made. The images or other third party material in this article are included in the article's Creative Commons license, unless indicated otherwise in a credit line to the material. If material is not included in the article's Creative Commons license and your intended use is not permitted by statutory regulation or exceeds the permitted use, you will need to obtain permission directly from the copyright holder. To view a copy of this license, visit http://creativecommons.org/ licenses/by/4.0/.

(C) The Author(s) 2021 